ARTICLE

https://doi.org/10.1038/s41467-019-09131-y

\title{
Ventral tegmental area astrocytes orchestrate avoidance and approach behavior
}

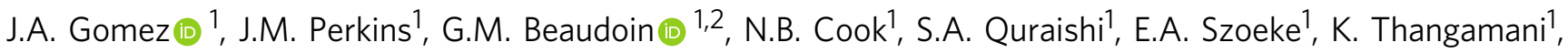 \\ C.W. Tschumi ${ }^{3}$, M.J. Wanat (1) ${ }^{1}$, A.M. Maroof ${ }^{1}$, M.J. Beckstead ${ }^{3}$, P.A. Rosenberg $\mathbb{B}^{4}$ \& C.A. Paladini ${ }^{1}$
}

The ventral tegmental area (VTA) is a heterogeneous midbrain structure, containing neurons and astrocytes, that coordinates behaviors by integrating activity from numerous afferents. Within neuron-astrocyte networks, astrocytes control signals from distinct afferents in a circuit-specific manner, but whether this capacity scales up to drive motivated behavior has been undetermined. Using genetic and optical dissection strategies we report that VTA astrocytes tune glutamatergic signaling selectively on local inhibitory neurons to drive a functional circuit for learned avoidance. In this circuit, astrocytes facilitate excitation of VTA GABA neurons to increase inhibition of dopamine neurons, eliciting real-time and learned avoidance behavior that is sufficient to impede expression of preference for reward. Loss of one glutamate transporter (GLT-1) from VTA astrocytes selectively blocks these avoidance behaviors and spares preference for reward. Thus, VTA astrocytes selectively regulate excitation of local GABA neurons to drive a distinct avoidance circuit that opposes approach behavior.

\footnotetext{
${ }^{1}$ University of Texas at San Antonio Neurosciences Institute, Department of Biology, University of Texas at San Antonio, San Antonio, TX 78249, USA.

${ }^{2}$ Department of Biology, Trinity University, San Antonio, TX 78212, USA. ${ }^{3}$ Aging \& Metabolism Research Program, Oklahoma Medical Research Foundation, Oklahoma City, OK 73104, USA. ${ }^{4}$ Department of Neurology and the F.M. Kirby Neurobiology Center, Boston Children's Hospital, Boston, MA 02115, USA. Correspondence and requests for materials should be addressed to C.A.P. (email: carlos.paladini@utsa.edu)
} 
T he ability to coordinate approach and avoidance actions in different environments can determine individual survival. Avoidance learning is an adaptive mechanism, but can become maladaptive and lead to the development and persistence of many specific anxiety disorders including post-traumatic stress and social anxiety disorders ${ }^{1}$. Dopamine signaling from the ventral tegmental area (VTA) mediates approach and avoidance learning ${ }^{2}$. However, the VTA is a heterogeneous structure containing astrocytes and neurons that receive mixed and distributed signals from various independent afferents conveying information for cues that drive both approach and avoidance ${ }^{3,4}$. For example, both aversive stimuli and reward expectation increase VTA GABA neuron firing and can inhibit dopamine neurons, leading to avoidance behaviors ${ }^{5-9}$. A mechanism capable of separating different signals underlying reward and aversion within the VTA is, therefore, necessary to appropriately coordinate approach and avoidance, respectively. By selectively affecting the activity of different neurons, VTA astrocytes may be positioned to coordinate avoidance and approach behaviors.

Astrocytes control glutamate availability at synapses of actively firing afferents primarily by the glutamate transporter, GLT-1 $\left(\right.$ EAAT2, Slc1a2) ${ }^{10}$. The contribution of glutamate transporters to signaling in neuron-astrocyte networks varies considerably among synapses and often changes depending on afferent activity, local ionic environment, and structure of the synapse ${ }^{10-14}$. Thus, rather than indiscriminately clearing glutamate, astrocytes are diverse regulators of neuronal processing that can delineate synapse-specific control to circuits in response to neuronal afferent activity $13-20$. Astrocytes express receptors and transporters that are activated by synaptically released neurotransmitters from neuronal afferents ${ }^{14,21-24}$. In response to afferent activity, astrocytes display a phasic increase in cytoplasmic $\mathrm{Na}^{+}$and $\mathrm{H}^{+}$, and a decrease in $\mathrm{K}^{+}$concentrations, among other ions ${ }^{12,25-28}$. The ensuing fast changes in the electrochemical gradient generate biophysical fluctuations that slow down astrocytic clearance of extracellular glutamate $e^{14,28-30}$. Thus, astrocytes are ideally suited to dynamically control glutamate at specific synapses throughout a wide concentration range ${ }^{31-33}$, thereby affecting postsynaptic neuronal activity and potentially driving behavior.

In the present study, we examined the role of astrocytes on VTA circuit activity and behavior. We found that VTA astrocytes (1) selectively facilitate glutamatergic excitation of local GABA neurons through a mechanism that requires astrocytic GLT-1; (2) generate a subsequent GABA neuron-dependent inhibition of dopamine neurons; and (3) consequently drive learned avoidance that is sufficient to block conditioned place preference for cocaine. Conditional mutants lacking GLT-1 in VTA astrocytes fail to express the tested avoidance behaviors, but maintain preference for reward. These experiments establish that VTA astrocytes selectively leverage glutamatergic excitation of local GABA neurons to dynamically define a privileged neuronastrocyte circuit underlying behaviors associated with aversion, but not reward.

\section{Results}

Astrocytes modulate excitation of VTA GABA neurons. To investigate the mechanism by which astrocytes regulate the circuitry of the VTA we obtained ex vivo recordings of VTA astrocytes (Fig. 1a). We first determined that neuronal afferent
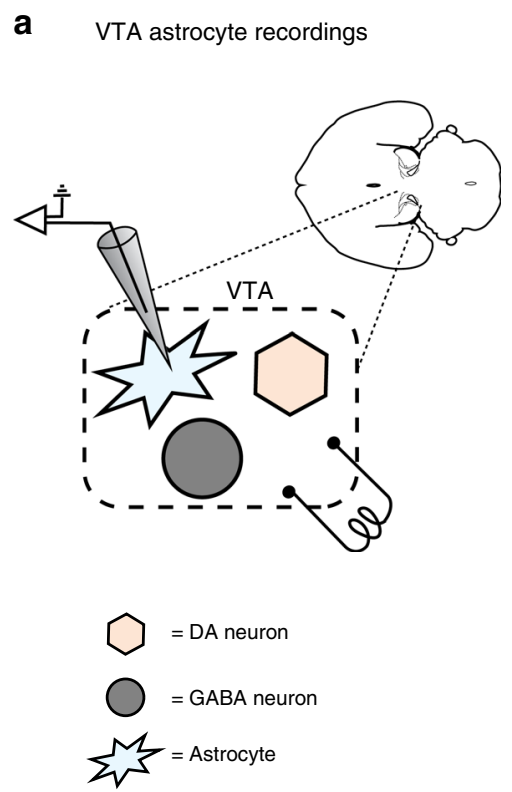

b

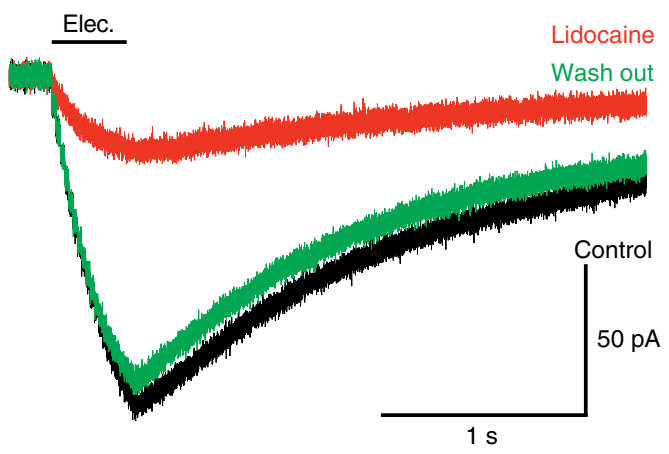

d

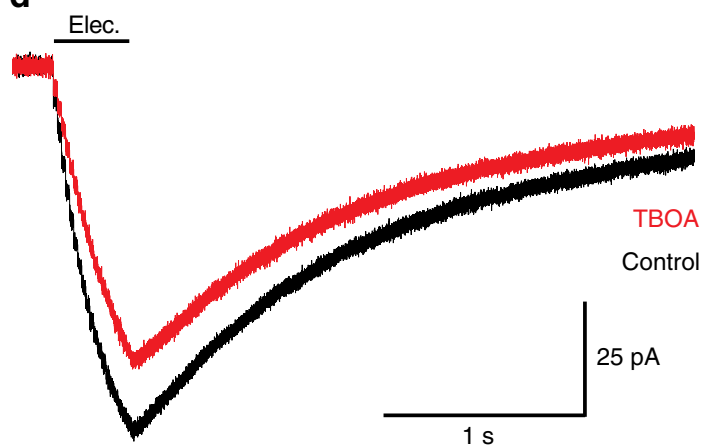

c

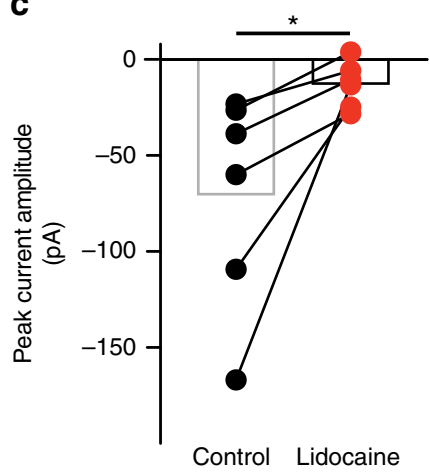

e

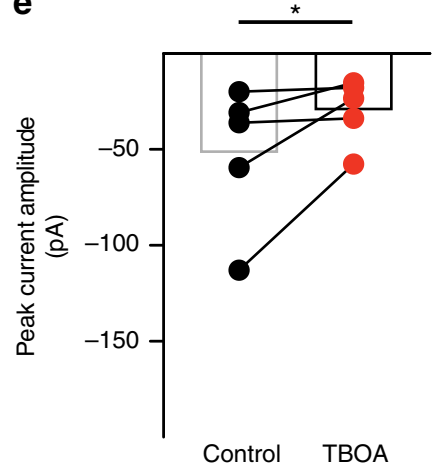

Fig. 1 Neuronal afferent activity changes glutamate transport in VTA astrocytes. a Schematic showing the microcircuitry of the VTA and recordings performed. b Recording from a VTA astrocyte showing the current generated by electrical stimulation (black; $50 \mathrm{~Hz}, 20$ pulses), the current remaining after blocking action potentials with $250 \mu \mathrm{M}$ lidocaine (red), and after washing out lidocaine (green). c Summary of the currents before (black) and after lidocaine application (red; paired $t$-test $t_{(5)}=2.679, P=0.04, n=6$ cells, 4 mice). d Current generated in a VTA astrocyte by electrical stimulation of afferents before (black) and after adding $15 \mu \mathrm{m}$ TFB-TBOA (red). e Summary of the currents generated by electrical stimulation in VTA astrocytes before (black) and after (red) addition of TFB-TBOA (paired t-test $\mathrm{t}_{(4)}=2.159, P=0.04, n=5$ cells, 5 mice). *: $P<0.05$ 
stimulation elicits action potential-dependent currents in VTA astrocytes (Fig. 1, and Supplementary Fig. 1). Electrical stimulation of the VTA during whole-cell voltage-clamp recordings of astrocytes resulted in a lidocaine-sensitive inward current (Fig. 1b, c). A significant portion of the inward current (35 $\pm 11 \%)$ was sensitive to the glutamate transporter blocker, TFB-TBOA $(15 \mu \mathrm{M})$, indicating that, similar to other reports ${ }^{14}$, neuronal afferent input activates glutamate receptors and glutamate transporter activity on VTA astrocytes (Fig. 1d, e, and Supplementary Fig. 1).

To mimic the activity of neuronal inputs on astrocytic glutamate transport we used an optogenetic strategy to selectively activate VTA astrocytes ${ }^{34}$. Similar to afferent activation of receptors on astrocytes ${ }^{14,25,30}$ (Supplementary Fig. 1), photoactivation of the light-sensitive opsin, channelrhodopsin (ChR2), results in a phasic increase in cytoplasmic $\mathrm{Na}^{+}$and $\mathrm{H}^{+}$, and a decrease in $\mathrm{K}^{+}$concentrations ${ }^{35,36}$. Therefore, owing to its cation permeability and time resolution ${ }^{35}$, ChR2 presents as an ideal tool to transiently influence astrocytic GLT-1 dynamics with a temporal fidelity capable of mimicking native astrocyte gradient dynamics ${ }^{28,29,37}$. ChR2 was expressed selectively in VTA astrocytes by injecting adeno-associated virus (AAV) to drive expression of ChR2 under the gfaABC1D promoter (gfaABC1D:: ChR2VTA; Fig. 2a, b, and Supplementary Fig. 2) ${ }^{38}$. We then confirmed that both neuronal afferent stimulation and optogenetic photoactivation of astrocytes similarly affect glutamate

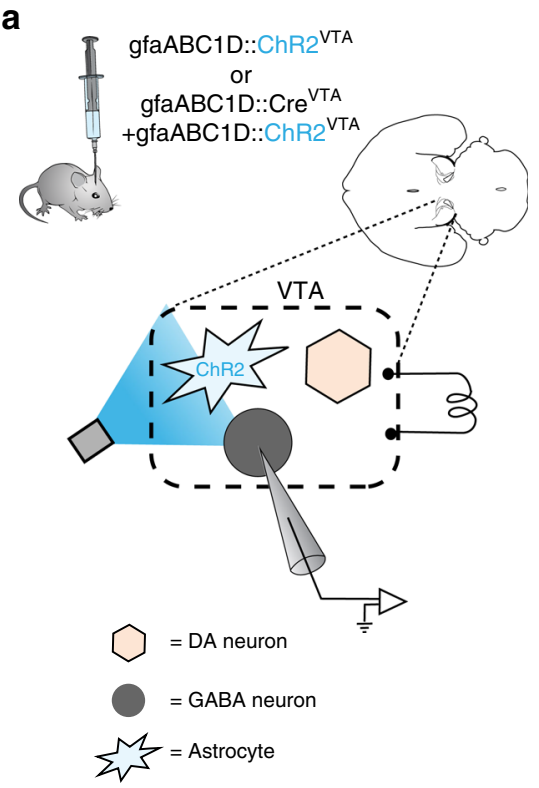

e

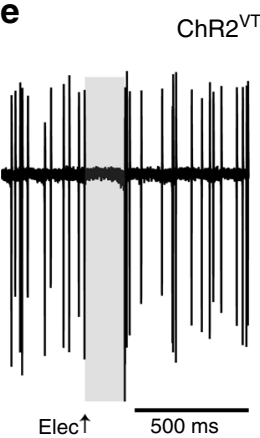

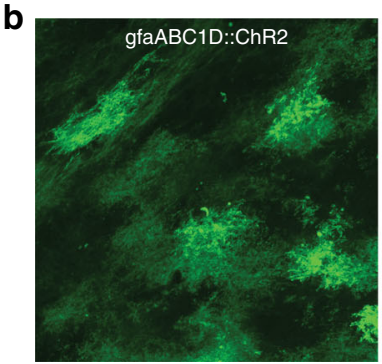

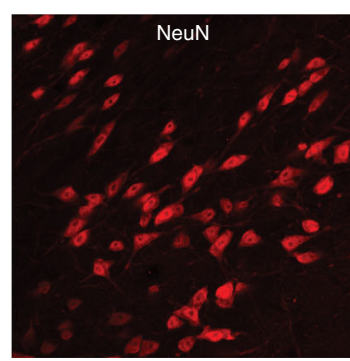

C

$$
\text { I }
$$

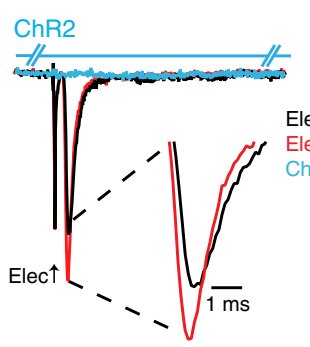

f
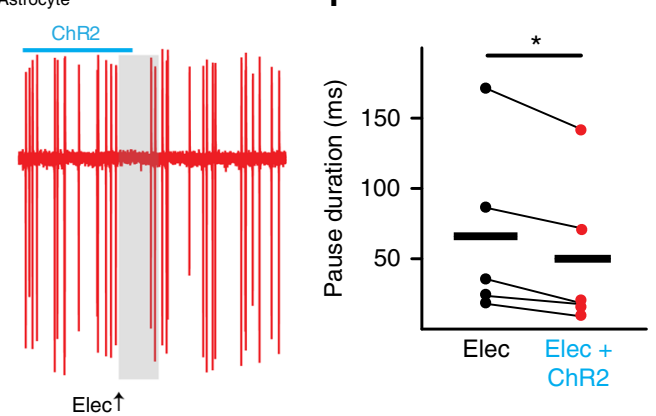

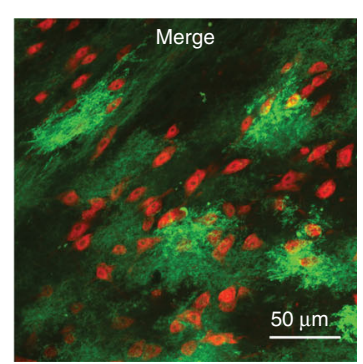

d

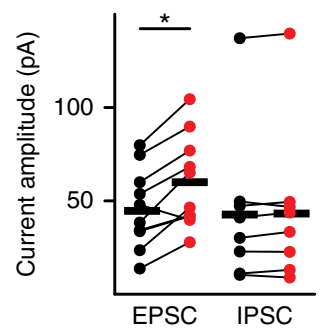

g $\mathrm{CHR} 2^{\text {VTA Astrocyte }}$
GLT-1 CKO ChR2

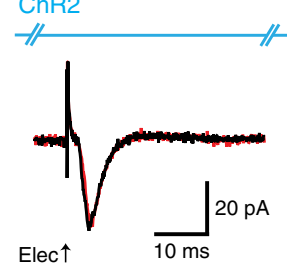

h

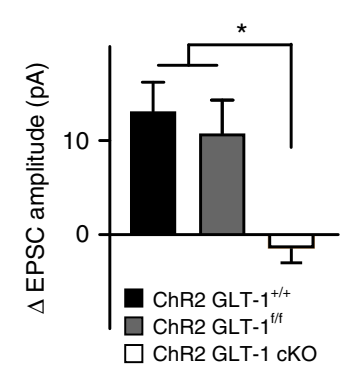

Fig. 2 Astrocytic GLT-1 increases the excitation of VTA GABA neurons. a Schematic showing the microcircuitry of the VTA and manipulations performed. b Immunocytochemistry images ( $\times 20$ objective) showing lack of co-localization between ChR2 (green, note characteristic astrocyte morphology) and NeuN (red) within VTA. c Example voltage-clamp recordings (left $V_{h}=-55 \mathrm{mV}$, right $V_{h}=+10 \mathrm{mV}$ ). The inward current (left), but not the outward current (right), increases when astrocytes are photoactivated (ChR2, blue hash marked horizontal line above traces) concurrently with electrical stimulation (Elec.) of neuronal afferents (red traces) in a GLT-1+/+ mouse. ChR2 photoactivation alone produces no response (blue trace; see Supplementary Fig. 8). d Summarized data from VTA GABA neurons where ChR2 stimulation on VTA astrocytes increases peak EPSC amplitude (GLT-1 $+/+$, paired t-test $t_{(9)}=4.457, P=0.016, n=10$ cells) without altering IPSCs (paired $t$-test $t_{(7)}=1.318, P=0.22, n=8$ cells, 4 mice). e Five overlaid traces of a cell-attached recording from a VTA GABA neuron. Electrical stimulation of neuronal afferents in the VTA (black traces) elicits a pause in firing activity (gray area). However, when ChR2 on VTA astrocytes (gfaABC1D::ChR2VTA) is photoactivated concurrently with electrical stimulation (red traces) the pause duration is decreased (see spikes within the gray area, which is pause duration of electrical stimulation alone). $\mathbf{f}$ Summarized data from GABA neurons demonstrating a decrease in pause duration (paired $t$-test $t_{(4)}=3.345, P=0.028, n=5$ cells, 2 mice) elicited by ChR2 stimulation on VTA astrocytes. $\mathbf{g}$ The increase in EPSC amplitude is absent in a GLT-1 cKOVTA Astrocyte mouse (GLT-1 f/f, gfaABC1D::CreVTA + gfaABC1D::ChR2VTA). h Summarized data comparing the change in the EPSC amplitude between GLT-1+/+ (GLT-1+/+, gfaABC1D::ChR2VTA), GLT-1/f/f (GLT-1/f/f, gfaABC1D:: ChR2 ${ }^{V T A}$ ), and GLT-1 cKOVTA Astrocyte mice (One-way ANOVA, $F_{(2,22)}=7.352, P=0.0036$; post-hoc Tukey's test GLT-1+/+ vs GLT-1/f $P>0.05$, GLT-1 $+/+$ vs GLT-1 cKOVTA Astrocyte $P<0.05$, GLT-1 ${ }^{f / f}$ vs GLT-1 cKOVTA Astrocyte $P<0.05, n=11, n=6, n=8$ cells, respectively). ${ }^{\star \star} p<0.01,{ }^{\star} P<0.05 ;$ Error bars indicate \pm SEM 
transporter-mediated currents in VTA astrocytes (Supplementary Fig. 3).

We investigated whether local GABA neurons are subject to modulation by VTA astrocytes. We found that during voltageclamp recordings of VTA GABA neurons (Supplementary Fig. $4 \mathrm{a}-\mathrm{e}$ ), electrically-stimulated excitatory postsynaptic currents (EPSCs) increased in peak amplitude when the electrical stimulation was paired with ChR2 photoactivation on VTA astrocytes $\left(\mathrm{GLT}-1^{\mathrm{f} / \mathrm{f}}, \mathrm{Elec}=22.0 \pm 3.7 \mathrm{pA}, \mathrm{Elec}+\mathrm{ChR} 2=32.4 \pm\right.$ $6.6 \mathrm{pA}$; paired $t$-test $t_{(5)}=3.147, P=0.025, n=6$ cells, 3 mice) with no effect on inhibitory postsynaptic currents (IPSCs) (GLT$1^{\mathrm{f} / \mathrm{f}}$, Elec $=44.8 \pm 18.1 \mathrm{pA}$, Elec $+\mathrm{ChR} 2=40.4 \pm 22.1 \mathrm{pA}$; paired $t$-test $t_{(4)}=0.8756, P=0.43, n=5$ cells, 3 mice; data not shown for GLT-1 ${ }^{\mathrm{f} / \mathrm{f}}$ mice). When the same cells were recorded in cellattached mode, electrical stimulation of all afferents in the brain slice (no blockers were present) elicited a net pause in firing. When the electrical stimulation was paired with photoactivation of astrocytes, the pause duration decreased, which is consistent with an increase in EPSC amplitude observed in voltage-clamp mode (Fig. 2e, f). Astrocyte photoactivation had no effect on the intrinsic properties of neurons (Supplementary Fig. 5).

Because astrocyte photoactivation with ChR2 could influence glutamate transport ${ }^{28,30,35,36}$, we next sought to determine whether VTA astrocytes increase excitation of local GABA neurons in a glutamate transporter-dependent manner. We drove a conditional knock out (cKO) by injecting an adeno-associated virus (AAV) into the VTA of GLT-1 floxed mice $\left(\mathrm{GLT}-\mathrm{1}^{\mathrm{f} / \mathrm{f}}\right)^{39}$ to restrict recombination and loss of GLT-1 to VTA astrocytes $\left(\right.$ GLT-1 ${ }^{\mathrm{f} / \mathrm{f}}$, AAV(gfaABC1D-Cre) $\rightarrow$ VTA; Fig. 2a, and Supplementary Fig. 6-7). The increase in EPSC amplitude elicited by astrocyte photoactivation was absent when GLT-1 was conditionally knocked out from VTA astrocytes (GLT-1 cKOVTA Astrocyte; Elec $=28.18 \pm 4.5 \mathrm{pA}, \mathrm{Elec}+\mathrm{ChR} 2=28.6 \pm 3.8$; paired $t$-test $t_{(7)}=0.3152, P=0.76, n=8$ cells, 3 mice; Fig. $2 \mathrm{~g}$ ), indicating that astrocyte activation increases EPSC amplitude in VTA GABA neurons via a mechanism that requires GLT-1 expression in astrocytes.

\section{Astrocytes modulate inhibition of VTA dopamine neurons.} Contrary to what we observed from GABA neurons, when we recorded from VTA dopamine neurons (Supplementary Fig. $4 \mathrm{f}-\mathrm{j}$ ), the IPSC peak amplitude increased, but the EPSC was unaffected, when electrical stimulation was paired with VTA astrocyte photoactivation $\left(\mathrm{GLT}-1^{\mathrm{f} / \mathrm{f}}\right.$ IPSC, Elec $=44.7 \pm 10.6 \mathrm{pA}$, Elec + ChR2 $=56.9 \pm 11.4$; paired $t$-test $t_{(6)}=4.243, P=0.0054$, $n=7$ cells, 2 mice, GLT- ${ }^{\text {f/f }}$ EPSC: Elec $=40.4 \pm 11.3$ pA, Elec + ChR2 $=42.8 \pm 12.4$; paired $t$-test $t_{(6)}=1.199, P=0.27, n=7$ cells, 2 mice; data not shown for GLT-1 ${ }^{\mathrm{f} / \mathrm{f}}$ mice). When the same dopamine cells were recorded in cell-attached mode, electrical stimulation of all afferents in the brain slice (no blockers present) also elicited a net pause in firing. However, when the electrical stimulation was paired with photoactivation of astrocytes, the pause duration increased (Fig. 3d, e), which is consistent with the increase in IPSC amplitude observed in voltage-clamp mode. The increase in IPSC amplitude was blocked when the glutamate transporter blocker, DHK $(300 \mu \mathrm{M})$, was added during the same recording (paired $t$-test $t_{(6)}=5.675, P=0.0013, n=7$ cells, 3 mice; Fig. 3f, g). Also, when GLT-1 was conditionally knocked out from VTA astrocytes, the IPSC amplitude on DA neurons was unaffected by astrocyte activation paired with electrical stimulation (GLT-1 cKOVTA Astrocyte; Elec $=51.7 \pm 9.5 \mathrm{pA}$, Elec + ChR2 $=52.4 \pm 10.2$; paired $t$-test $t_{(7)}=0.2926, P=0.77, n=8$ cells, 2 mice; Fig. 3h). Importantly, the effect of GLT-1 manipulation was dependent on active synaptic input since photoactivation of VTA astrocytes without electrical stimulation of neuronal afferents had no effect on either VTA dopamine or GABA neurons (Figs. 2c, 3b, and Supplementary Fig. 8). Thus, astrocytes use a GLT-1-dependent mechanism to increase both excitation in GABA neurons and inhibition in dopamine neurons, but only during concurrent activity of neuronal afferents.

GABA neurons mediate increased dopamine neuron inhibition. Given the surprising result of a glutamate transporter mediating the increase of an outward current in dopamine neurons, we next determined whether the outward current was indeed an IPSC mediated by an inhibitory input. We tested the sensitivity of the outward current to the $\mathrm{GABA}_{\mathrm{A}}$ receptor antagonist, bicuculline $(30 \mu \mathrm{M})$. Both the electrically-induced IPSC, and the peak amplitude increase elicited by pairing electrical stimulation with photoactivation of astrocytes, were abolished by addition of bicuculline, indicating that all outward currents were mediated by $\mathrm{GABA}_{\mathrm{A}}$ receptor activation (paired $t$-test $t_{(4)}=3.682, P=0.0015, n=5$ cells, 3 mice; Fig. $4 \mathrm{a}-\mathrm{c}$ ). However, only the increase in IPSC amplitude, not the electrically-elicited outward current per se, was sensitive to glutamate receptor antagonism (10 NBQX $+100 \mathrm{AP} 5$, paired $t$-test $t_{(7)}=3.101, P=0.0173, n=8$ cells, 4 mice; Fig. $4 d$, e). Since (1) ChR2 activation increased the EPSC amplitude on VTA GABA neurons, and (2) the increased IPSC on dopamine neurons was dependent on glutamate receptor activation, dopamine neurons may be inhibited via a circuit mechanism involving depolarization of GABA neurons.

To directly test whether local GABA neurons mediated the effects of astrocyte activation on dopamine neuron inhibition, we optogenetically hyperpolarized VTA GABA neurons (VGATCre.: eNpHR ${ }^{\mathrm{VTA}}$ ) during photoactivation of ChR2 on VTA astrocytes (gfaABC1D::ChR2VTA; Fig. 5a, b, and Supplementary Fig. 9). Optogenetic hyperpolarization of VTA GABA neurons prevented the increase in IPSC amplitude, recorded from neighboring dopamine neurons, that resulted from combining electrical stimulation with optogenetic activation of VTA astrocytes during the same recordings (unpaired $t$-test $t_{(18)}=3.961, P=0.0009$, electrical + ChR2: $n=7$ cells, 4 mice, electrical + ChR2 + NpHR: $n=13$ cells, 6 mice; Fig. $5 c$, d).

VTA astrocytes drive real-time avoidance mediated by GABA. Previous studies demonstrate that direct optogenetic depolarization of VTA GABA neurons inhibits the activity of neighboring dopamine neurons and elicits avoidance behavior ${ }^{6,7}$. Our recordings demonstrate that VTA astrocytes can increase the response of GABA neurons to active excitatory afferent input, leading to increased inhibition of dopamine neurons. We, therefore, aimed to determine whether ChR2 activation of astrocytes would directly drive avoidance behavior. Extending our same optogenetic strategy to behavioral assays, we found that photoactivation of VTA astrocytes induced real-time avoidance in control mice in a real-time place preference (RTPP) assay $\left(\mathrm{GLT}-1^{+/+}\right.$, paired $t$-test $t_{(17)}=11.56, P=0.0001, n=18$ mice; Fig. 6b, e; Supplementary Fig. 10, and Supplementary Movie 1). The effect of astrocyte activation was reversible since mice could be manipulated to avoid either side of the chamber by changing which side was paired with laser stimulation (Fig. 6b, c). The avoidance behavior was also dependent on astrocytic GLT-1 since conditional knockout restricted to VTA astrocytes (GLT-1 cKOVTA Astrocyte, gfaABC1D::ChR2 ${ }^{\text {VTA }}$ ) eliminated the real-time avoidance elicited by photoactivating VTA astrocytes (paired $t$-test $t_{(10)}=0.1399, P=0.98, n=11$ mice; Fig. 6d, e).

We then confirmed that GABA neuron depolarization is necessary for eliciting real-time place avoidance by optogenetically hyperpolarizing VTA GABA neurons concurrently with 


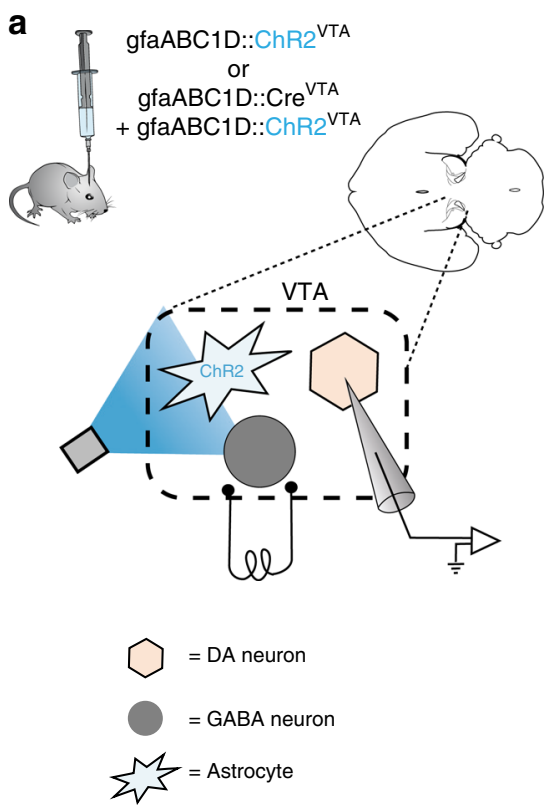

b

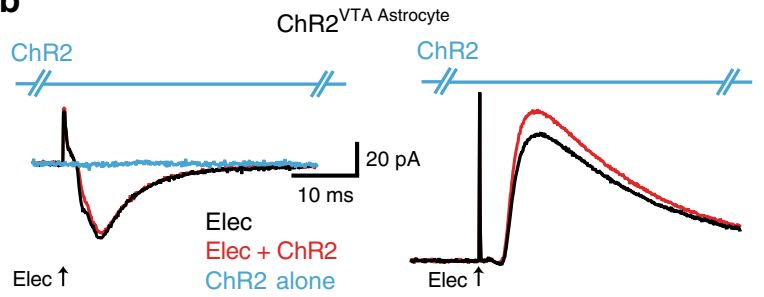

d
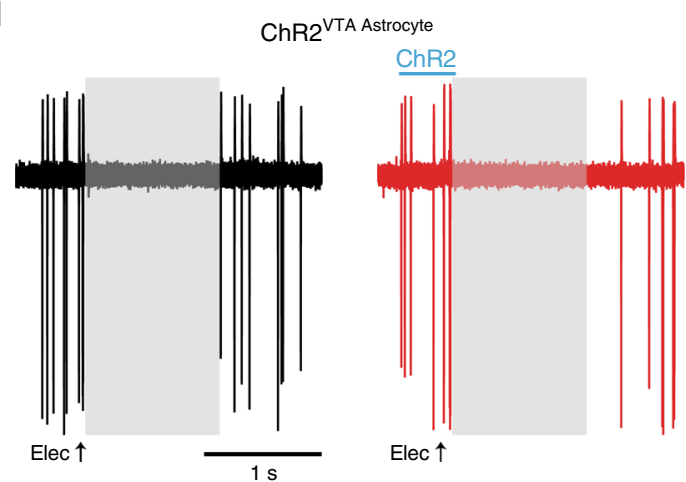

$\mathbf{f}$

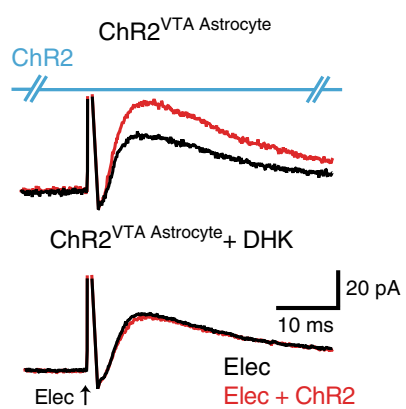

g

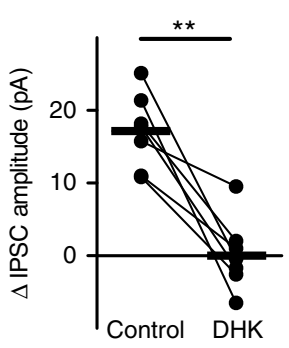

h

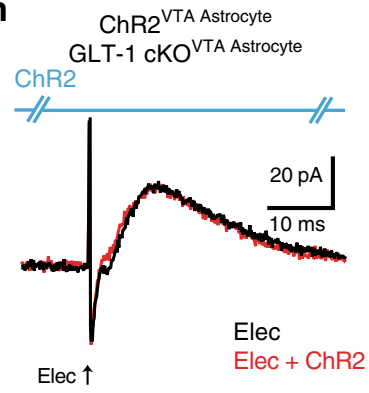

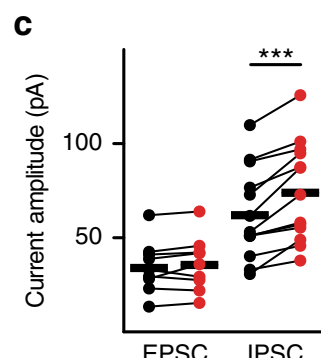

e
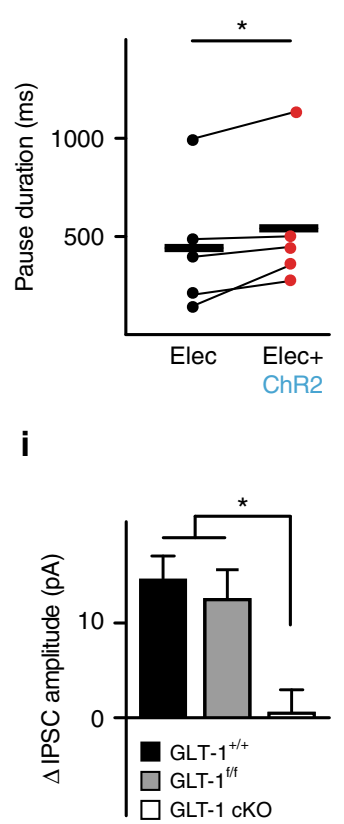

Fig. 3 Astrocytic GLT-1 increases inhibition of VTA dopamine neurons. a Schematic showing the microcircuitry of the VTA and manipulations performed. b Example voltage-clamp recordings (left $V_{h}=-55 \mathrm{mV}$, right $\mathrm{V}_{\mathrm{h}}=+10 \mathrm{mV}$ ). For dopamine neurons, the outward current (right), not the inward current (left), increases when ChR2 is photoactivated concurrently with electrical stimulation (Elec.) of neuronal afferents (red traces) in a GLT-1+/+ mouse. Photoactivation of ChR2 alone elicits no response (blue trace, and see Supplementary Fig. 8). c Summarized data from VTA dopamine neurons where ChR2 stimulation on VTA astrocytes increases peak IPSC amplitude (GLT-1+/+, paired $t$-test $t_{(12)}=5.894, P=0.0001, n=13$ cells, 8 mice) without altering EPSCs (paired $t$-test $t_{(9)}=1.129, P=0.28, n=10$ cells, 8 mice). $\mathbf{d}$ Nine overlaid traces of a cell-attached recording and $\mathbf{e}$ summarized data from VTA dopamine neurons demonstrating an increase in pause duration (paired $t$-test $t_{(4)}=2.815, P=0.04, n=5$ cells, 2 mice) when ChR2 stimulation on VTA astrocytes is delivered concurrently with electrical stimulation of neuronal afferents (red traces; gray areas are the pause duration following electrical stimulation alone). $\mathbf{f}, \mathbf{g}$ The increase in IPSC amplitude (red trace, top) is abolished by pharmacologically blocking GLT-1 with $300 \mu$ M DHK (bottom traces). $\mathbf{h}$ When GLT-1 is conditionally knocked out from VTA astrocytes there is no change in IPSC caused by activating ChR2 on astrocytes. $\mathbf{i}$ Summarized data comparing the change in IPSC amplitude between GLT-1+/+ (GLT-1+/+, gfaABC1D::ChR2VTA), GLT-1/f/f (GLT-1/f, gfaABC1D::ChR2VTA), GLT-1 $1^{f / f}$, and GLT-1 cKOVTA Astrocyte mice (One-way ANOVA, $F_{(2,27)}=7.732, P=0.0022$; post-hoc Tukey's test GLT-1 ${ }^{+/+}$vs GLT-1 $1^{f / f} P>0.05$, GLT-1+/+ vs GLT-1 cKOVTA Astrocyte $P<0.05$, GLT-1 ${ }^{\mathrm{f} / \mathrm{f}}$ vs GLT-1 cKOVTA Astrocyte $P<0.05, n=15, n=7, n=8$ cells, respectively). ${ }^{\star \star} P<0.01,{ }^{\star} P<0.05 ;$ Error bars indicate \pm SEM

ChR2 activation of VTA astrocytes. Similar to the increase in IPSC amplitude in dopamine neurons, real-time avoidance elicited by photoactivation of VTA astrocytes from VGATCre:: NpHRVTA mice was blocked by concurrently hyperpolarizing local GABA neurons (paired $t$-test $t_{(6)}=3.642$, eNpHR $+\mathrm{ChR} 2$; $P=0.01, n=7$ mice; Fig. 6g, h, and Supplementary Fig. 9). Therefore, astrocyte activation reliably induced real-time avoidance behavior through astrocytic GLT-1-dependent and GABA neuron depolarization-dependent mechanisms that are consistent with the circuit pathway established in our ex vivo experiments.

Astrocytes are required for learned avoidance not approach. Because of the VTA's well-known involvement in cue-outcome associative learning, we next sought to determine if astrocyte manipulation-evoked avoidance was sufficient to serve as a motivationally relevant signal in an associative learning context. The place conditioning paradigm is particularly well-suited for examining approach or avoidance since it makes use of the fact that mice will readily learn to approach or avoid distinct environments previously paired with either rewarding or aversive events, respectively ${ }^{40}$. We implemented a conditioned place avoidance (CPA) paradigm using photoactivation of VTA astrocytes as a conditioning stimulus paired to a contextual cue (chamber) during conditioning sessions. Astrocyte activation was sufficient to elicit learned avoidance (gfaABC1D::ChR2VTA; GLT$1^{\mathrm{f} / \mathrm{f}}$ : paired $t$-test $t_{(9)}=3.604, P=0.0057, n=10$ mice; Fig. $7 \mathrm{~b}, \mathrm{~d}$; and Supplementary Fig. 10), while GLT-1 cKO restricted to VTA astrocytes eliminated expression of avoidance learning during 


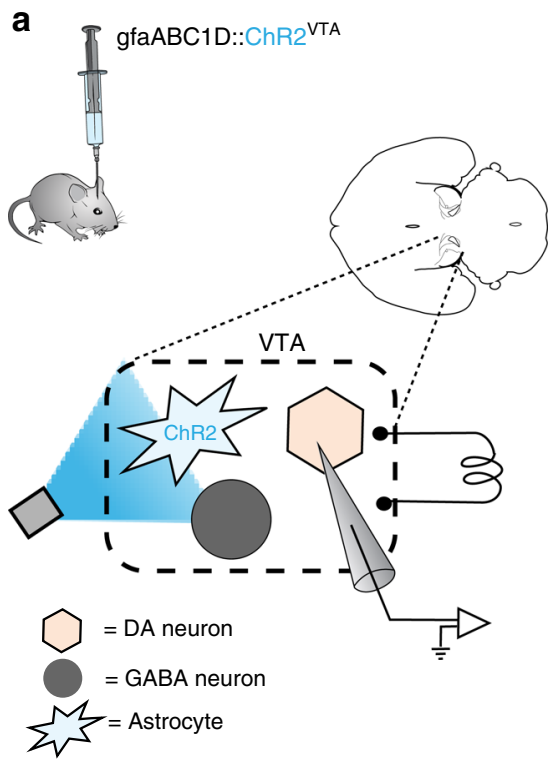

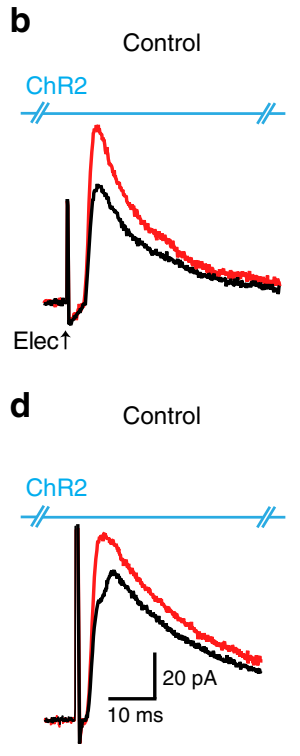

Elec $\uparrow$

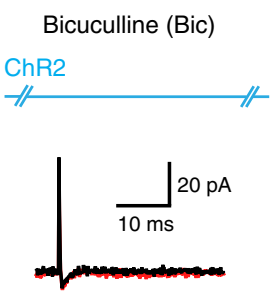

Elec $\uparrow$

$\mathrm{NBQX}+\mathrm{AP5}$

(Ant)

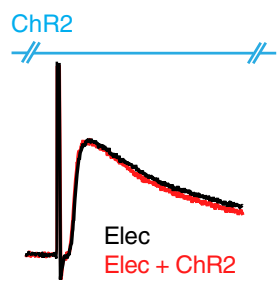

Elec $\uparrow$

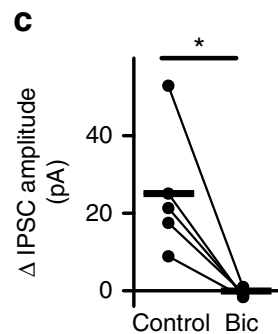

e

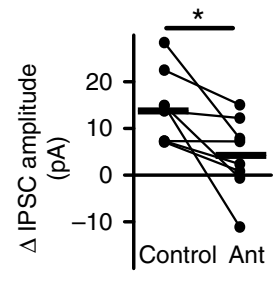

Fig. 4 Increase in dopamine neuron inhibition is dependent on glutamate receptor activation. a Schematic showing the microcircuitry of the VTA and manipulations performed. $\mathbf{b}$ On the left is a voltage-clamp $\left(V_{h}=+10 \mathrm{mV}\right)$ recording from a VTA dopamine neuron where pairing electrical simulation

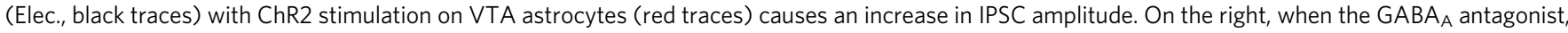
bicuculline $(30 \mu \mathrm{M})$, is added during the same recording the IPSC is abolished. c Data summarizing the effect of bicuculline. d The increase in IPSC amplitude was eliminated by glutamate receptor antagonists ( $10 \mu \mathrm{M} \mathrm{NBQX}+100 \mu \mathrm{M} \mathrm{AP-5)}$ with no effect on the amplitude of the IPSC elicited by electrical stimulation alone (black traces) (paired $t$-test, $P=0.99, n=8$ cells, 4 mice). e Data summarizing the effect of glutamate receptor antagonists. ${ }^{\star} P<0.05$

a

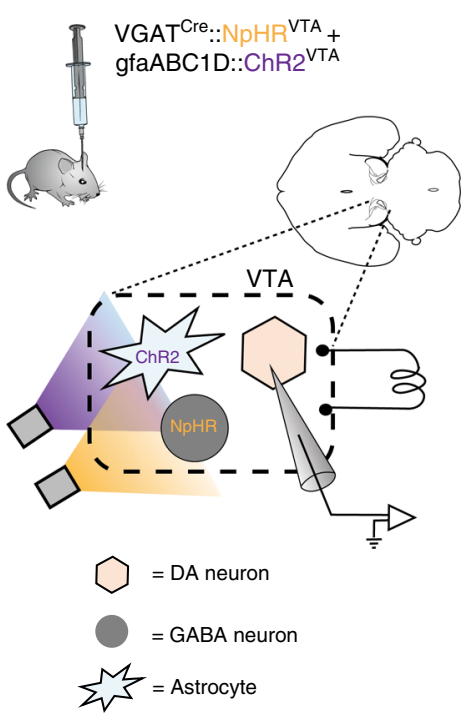

b

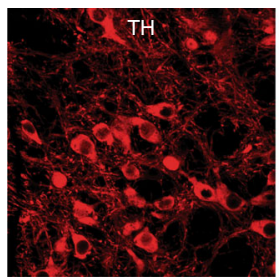
Ventral tegmental area (VTA)
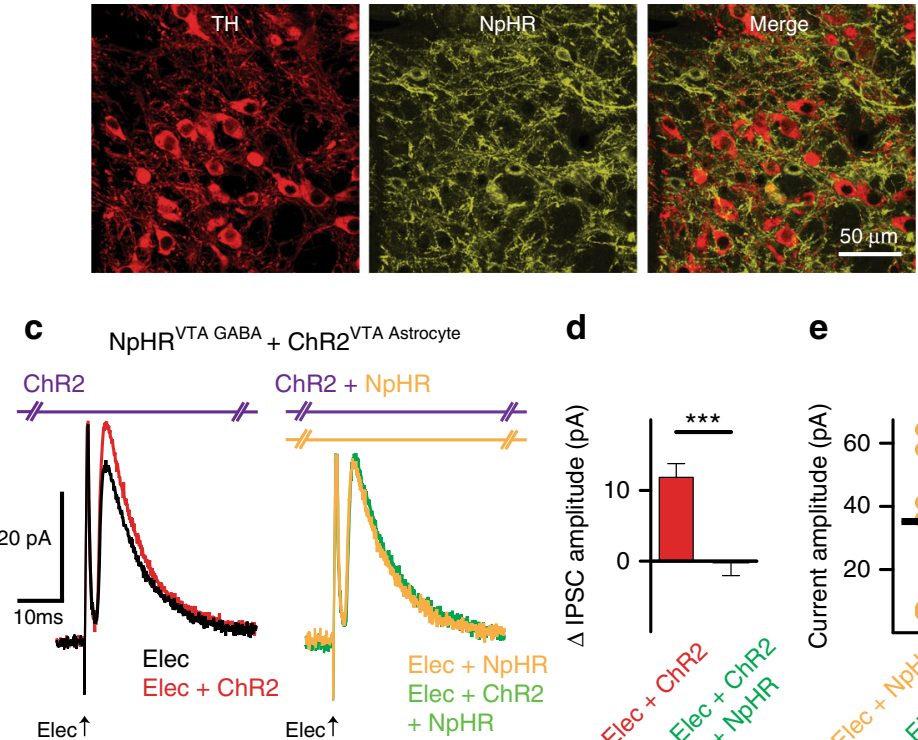

d

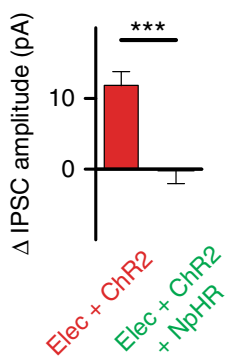

e

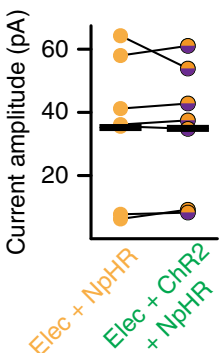

Fig. 5 Astrocyte-dependent increase in dopamine neuron inhibition is mediated by VTA GABA neurons. a Experimental manipulation. b Immunocytochemistry demonstrating TH positive cells (red) and eNpHR expression (yellow). On the right are the two images merged together. $\mathbf{c}$ Voltageclamp traces $\left(\mathrm{V}_{\mathrm{h}}=+10 \mathrm{mV}\right.$ ) from a VTA dopamine neuron. ChR2 stimulation during electrical stimulation (Elec + ChR2, red trace; ChR2 stimulated at $405 \mathrm{~nm}$ to minimize activation of eNpHR) increases the IPSC amplitude relative to electrical stimulation alone (Elec, black trace). When ChR2 on astrocytes is activated concurrently with eNpHR activation on GABA neurons during electrical stimulation (Elec $+\mathrm{ChR2}+\mathrm{eNpHR}$, green trace) the increase in IPSC amplitude was eliminated. Electrical stimulation with GABA neuron hyperpolarization alone (Elec + eNpHR, yellow trace) has no difference relative to electrical stimulation along with ChR2 on astrocytes and eNpHR activation on GABA neurons (Elec $+\mathrm{ChR2}+\mathrm{eNpHR}$, green trace). d The increase in IPSC amplitude when ChR2 was photoactivated with electrical stimulation (Elec + ChR2, red) was blocked when GABA neurons were simultaneously hyperpolarized (Elec + eNpHR + ChR2, green). e IPSC amplitudes during electrical stimulation with GABA neuron hyperpolarization $\left(\right.$ Elec + eNpHR, yellow) were not changed when ChR2 stimulation was added (Elec + eNpHR + ChR2, purple-yellow; paired $t$-test $t_{(6)}=0.1247, P=0.90$, $n=7$ cells, 4 mice). ${ }^{\star \star \star} P<0.001$; Error bars indicate \pm SEM 


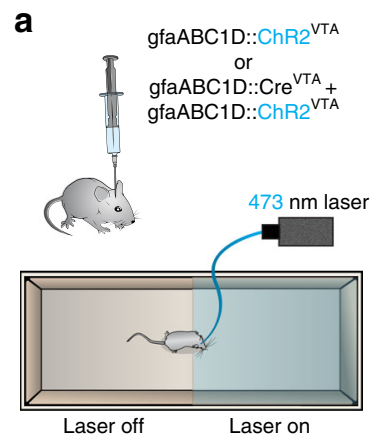

C

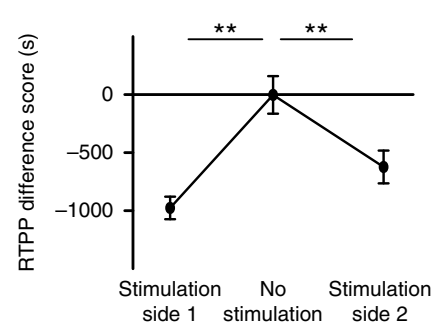

f

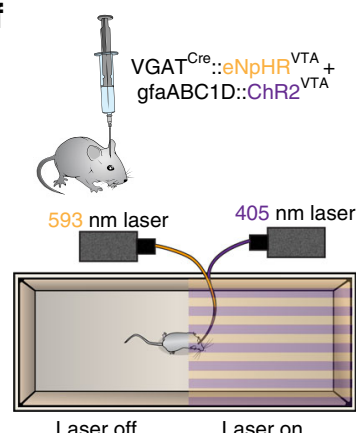

b

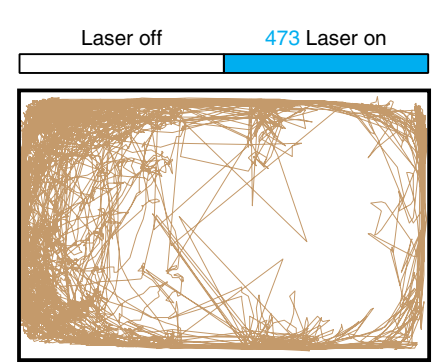

d

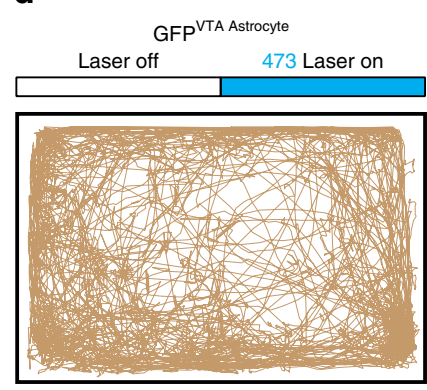

Real-time place preference $\mathrm{ChR} 2^{\text {VTA Astrocyte }}$
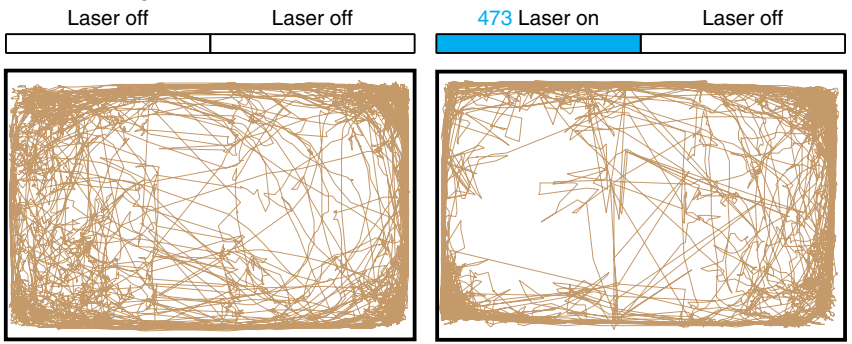

e

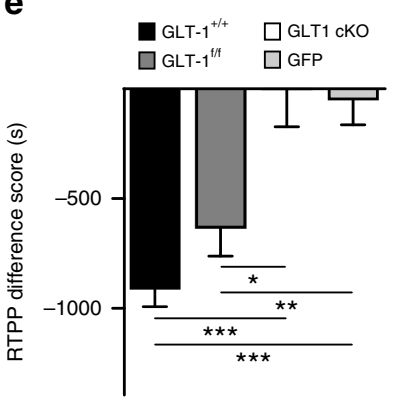

h

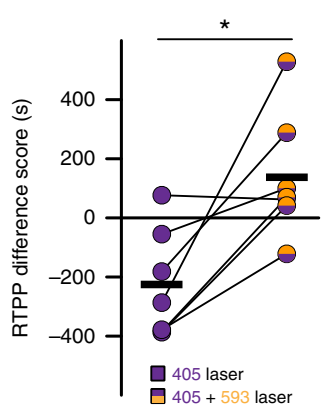

Fig. 6 Real-time avoidance is dependent on VTA astrocytes and mediated by local GABA neurons. a Experimental manipulations for panels b-e. $\mathbf{b}$ Three track plots from one ChR2VTA Astrocyte mouse (GLT-1+/+, gfaABC1D::ChR2VTA). (left) The mouse avoided the side of the chamber where ChR2 was stimulated in the VTA ( $473 \mathrm{~nm}$ Laser on). When the laser was left off both sides (Laser off) the mouse showed no avoidance. (right) With laser stimulation on the opposite side, the mouse then avoided the opposite side. c Summarized data showing avoidance of the laser-paired side (Repeated Measures ANOVA, $F_{(1.413,11.3)}=18.43, P=0.0006$; post-hoc Tukey's test, stimulation side 1 vs no stimulation $P<0.05$, stimulation side 1 vs stimulation side $2 P>$ 0.05 , Stimulation side 2 vs no stimulation $P<0.05, n=9$ mice). $\mathbf{d}$ (left) Track plot from a mouse expressing GFP on VTA astrocytes (GFPVTA Astrocyte; GLT-1 $1^{f / f}$, gfaABC1D::GFPVTA) shows no avoidance. (Right) track plot from a mouse expressing ChR2 on, but with GLT-1 conditionally knocked out from, VTA astrocytes (GLT-1 cKOVTA Astrocyte; GLT-1 ${ }^{f / f}$, gfaABC1D::ChR2VTA + gfaABC1D::CreVTA) showing no avoidance. e Summarized data showing avoidance elicited by ChR2 activation in GLT-1 ${ }^{+/+}$and GLT-1 $1 / f$ mice. However, loss of GLT-1 from VTA astrocytes eliminates avoidance (One-way ANOVA, $F_{(3,49)}=16.11, P=0.0001$; post-hoc Tukey's test, GLT-1 ${ }^{f / f}(n=10)$ relative to: GLT-1 cKOVTA Astrocyte $P<0.05, \mathrm{GLT}-1^{+/} /+P>0.05(n=18)$, GFP $P<0.05(n=14)$; cKOVTA Astrocyte $(n=11)$ relative to: GLT-1+/+ $P>0.05$, GFP $P<0.05 ;$ GLT-1+/+ $P<0.05)$. f Experimental manipulations for panels g-h. g Track plots from a VGATCRE::eNpHRVTA mouse with ChR2 stimulation on VTA astrocytes (eNpHRVTA GABA + ChR2VTA Astrocyte). Avoidance observed during ChR2 stimulation (left; ChR2 stimulated at $405 \mathrm{~nm}$ to minimize activation of eNpHR) was blocked when astrocytes were photoactivated concurrently with GABA neuron hyperpolarization (right). $\mathbf{h}$ Summarized data demonstrating GABA neuron hyperpolarization blocks avoidance elicited by ChR2 on VTA astrocytes. ${ }^{\star \star} P<0.01,{ }^{\star} P<0.05$; Error bars indicate \pm SEM

the assay (GLT-1 cKOVTA Astrocyte, gfaABC1D::ChR2VTA: paired $t$-test $t_{(11)}=0.7296, P=0.48, n=12$; Fig. $\left.7 \mathrm{c}, \mathrm{d}\right)$. Thus, VTA astrocytes mediate associative learning of aversive cues through a mechanism that requires expression of astrocyte GLT-1.

When a cue predicts both reward and aversion, dopamine neurons' activity responses reflect the integrated value of both outcomes ${ }^{9}$, indicating a convergence of information at or before the level of the dopamine neuron. As a step in establishing astrocytes as functional elements within the VTA approachavoidance circuit, we sought to disambiguate whether GLT-1 on astrocytes is positioned within a functional pathway specific to avoidance, or a common pathway that mediates expression of both avoidance and preference. If astrocytes mediate a common pathway, absence of astrocyte GLT-1 would be expected to abolish expression of both approach and avoidance. To dissect pathway specificity or generality, we used a conditioned place preference (CPP) paradigm to pair a contextual cue with a conditioning stimulus that contained both a strong reward (cocaine, $15 \mathrm{mg} / \mathrm{kg}$ ) and aversion (photoactivation of VTA astrocytes). Consistent with prior work $^{41}$, cocaine alone drove robust approach in the CPP assay (GLT-1 ${ }^{+1+}$ eYFP: paired $t$-test $\mathrm{t}_{(8)}=3.735, n=9 ; \quad P=0.0057$; not shown). However, when 
a

VTA astrocyte ChR2 conditioned place preference (CPP)
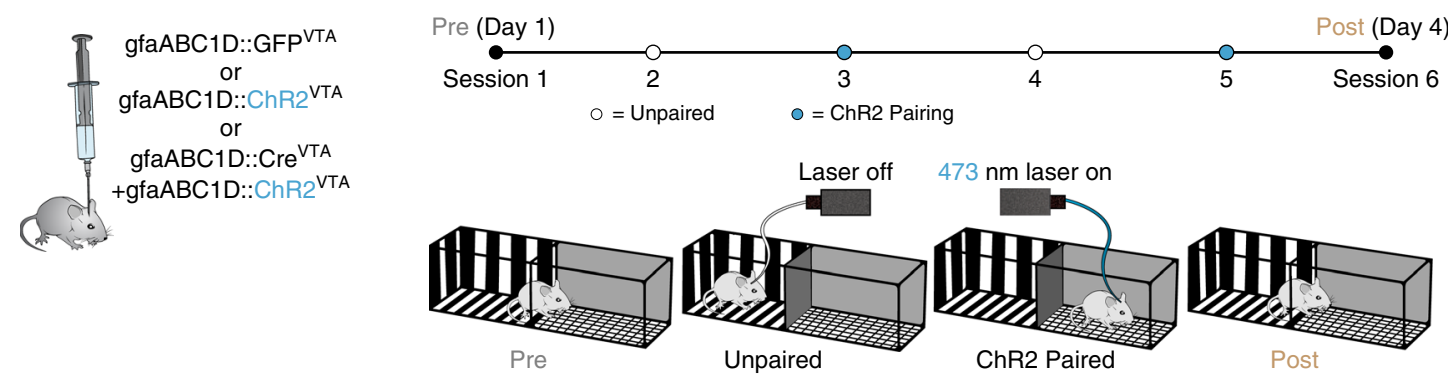

b $\mathrm{ChR} 2^{\text {VTA Astrocyte }}$

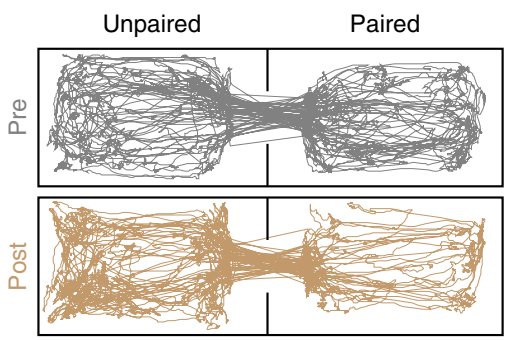

e
C
Unpaired

ChR2 $2^{\text {VTA Astrocyte }}$ d

d GLT-1 cKO VTA Astrocyte
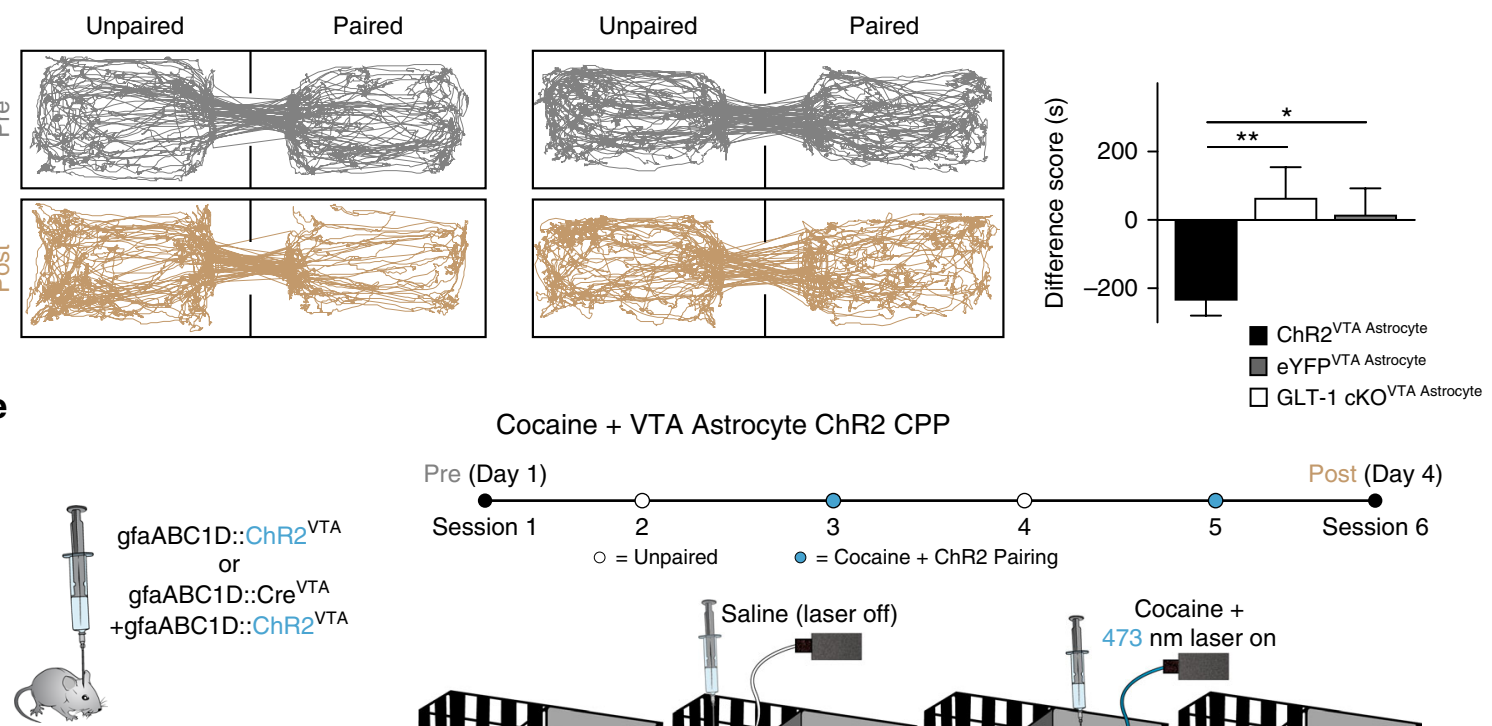

Cocaine + VTA Astrocyte ChR2 CPP

Post
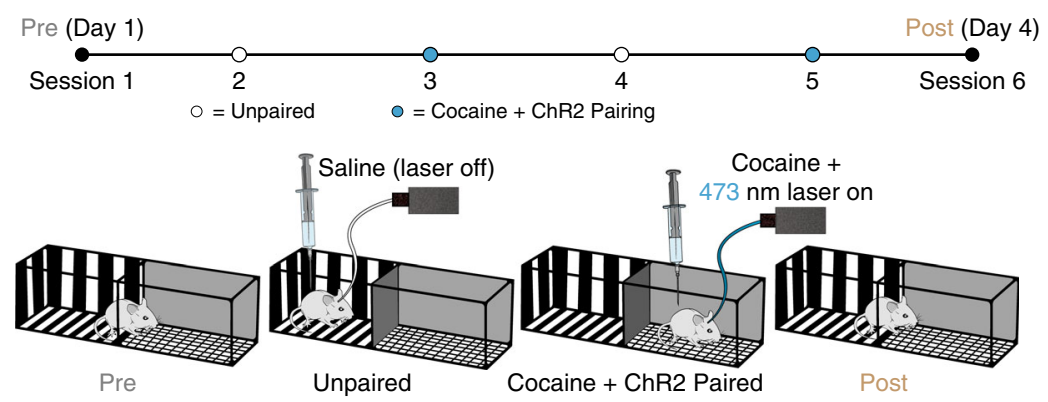

f

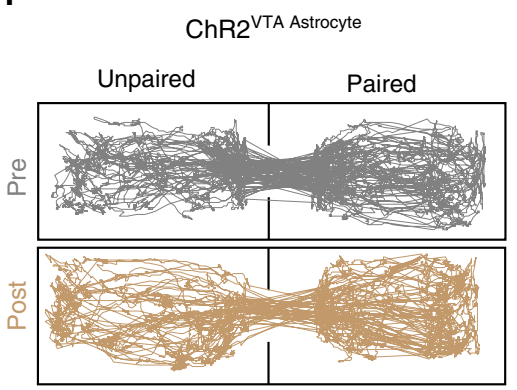

g
ChR2 ${ }^{\text {VTA Astrocyte }}$

GLT-1 cKOVTA Astrocyte

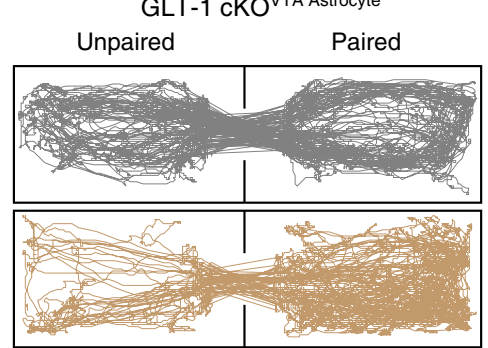

h

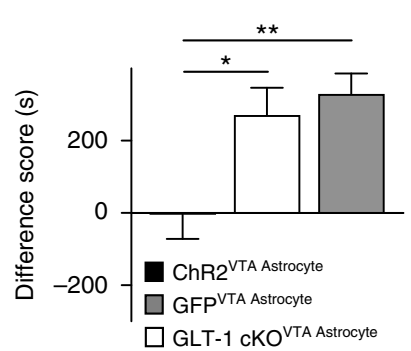

Fig. 7 VTA Astrocytes mediate learned avoidance and prevent learned preference for reward. a Experimental design and manipulations for panels b-d. b Track plots showing that conditioning with ChR2 photoactivation on VTA astrocytes elicits learned avoidance in a GLT-1+/+ mouse (gfaABC1D:: ChR2 ${ }^{V T A}$ ). c The learned avoidance is absent in a mouse where GLT-1 was removed from VTA astrocytes (gfaABC1D::ChR2VTA, GLT-1 cKOVTA Astrocyte). d Comparing the difference scores in mice expressing either ChR2 (GLT-1+/+ and GLT-1//f, GLT-1 cKOVTA Astrocyte) or GFP (gfaABC1D::ChR2eYFP) in VTA astrocytes. e Experimental design and manipulations for panels $\mathbf{f}-\mathbf{h}$. $\mathbf{f}$ Track plots showing astrocyte activation paired with cocaine administration does not elicit either learned avoidance or preference in a GLT-1/ff mouse (gfaABC1D::ChR2 VTA). $\mathbf{g}$ However, when GLT-1 is removed from VTA astrocytes (gfaABC1D::ChR2VTA, GLT-1 cKOVTA Astrocyte) a mouse displays robust learned preference for VTA astrocyte ChR2 photoactivation paired with cocaine administration. $\mathbf{h}$ Comparing the difference scores in mice expressing either ChR2 (GLT-1 ${ }^{+/+}$and GLT-1 ${ }^{f / f}$, GLT-1 cKOVTA Astrocyte) or GFP (gfaABC1D:: ChR2 ${ }^{\mathrm{eYFP}}$ ) in VTA astrocytes. ${ }^{\star \star} P<0.01,{ }^{\star} P<0.05$; Error bars indicate \pm SEM 
cocaine was paired with ChR2 activation on VTA astrocytes during the conditioning sessions, both the preference for cocaine and the avoidance of ChR2 photoactivation were abolished (gfaABC1D::ChR2 VTA: paired t-test $t_{(7)}=0.7588, P=0.47, n=8$ mice, Fig. $7 f$, h, and Supplementary Fig. 10). Since expression of CPA was mediated by GLT-1 on VTA astrocytes we next sought to determine whether cocaine CPP was also dependent on VTA astrocyte GLT-1 expression. However, preference for cocaine remained normal even when cocaine was paired with photoactivation of VTA astrocytes during conditioning in GLT-1 $\mathrm{cKO}^{\mathrm{VTA}}$ Astrocyte mice (One-way ANOVA, $\mathrm{F}_{(2,33)}=8.295, P=$ 0.0012; post-hoc Tukey's test eYFP vs ChR2 $P>0.05$, eYFP vs GLT-1 cKOVTA Astrocyte $P<0.05$, ChR2 vs cKOVTA Astrocyte $P>$ 0.05; ChR2: $n=15$, eYFP: $n=14$, GLT- 1 cKOVTA Astrocyte: $n=7$; Fig. $7 \mathrm{~g}, \mathrm{~h}$, and Supplementary Fig. 10). Thus, although CPA requires GLT-1 expression in VTA astrocytes and can suppress the expression of learned approach, CPP for cocaine is independent of astrocytic GLT- 1 in the VTA.

VTA astrocytic GLT-1 is necessary for open field avoidance. To determine the impact of VTA astrocytic GLT-1 function on endogenous behavior we tested whether selective deletion of GLT-1 in VTA astrocytes is sufficient to skew an animal's natural behavior in an open field test as an ethological assay that indexes approach-avoidance conflict. Mice with GLT-1 cKO restricted to
VTA astrocytes (GLT-1 cKOVTA Astrocyte) displayed a decreased tendency to remain in the peripheral zone near the walls of the chamber, and increased tendency to explore the center of the open field, relative to littermate controls (unpaired $t$-test $t_{(16)}=$ 2.449, $P=0.026, n=9$ mice; Fig. 8b, c). Disrupted approachavoidance behavior in GLT-1 cKOVTA Astrocyte mice indicates that VTA astrocytes are an integral component of a circuit within the VTA capable of coordinating approach and avoidance behaviors.

\section{Discussion}

Our experiments identify, through a series of genetic and optogenetic strategies in slice and in vivo, a specialized VTA microcircuit that is regulated by astrocytes to drive avoidance behavior. We observed that local astrocytes within the VTA circuit modulate glutamate availability via GLT-1 to dynamically regulate active GABAergic inhibition of dopamine neurons (Fig. 9). As a result, VTA astrocytes induced robust, time-locked behavioral avoidance de novo. Rapid manipulation of astrocyte glutamate transport in the VTA served as a powerful stimulus that, when paired with cocaine, was sufficient to impede behavioral expression of cocaine CPP. Thus, despite cocaine's many systemic effects, local astrocyte manipulation within the VTA was sufficient to override expression of preference for a highly salient cocaine-associated cue. These experiments suggest that midbrain

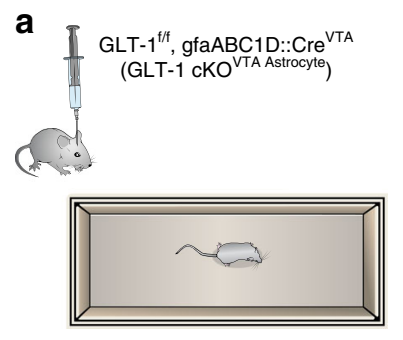

b

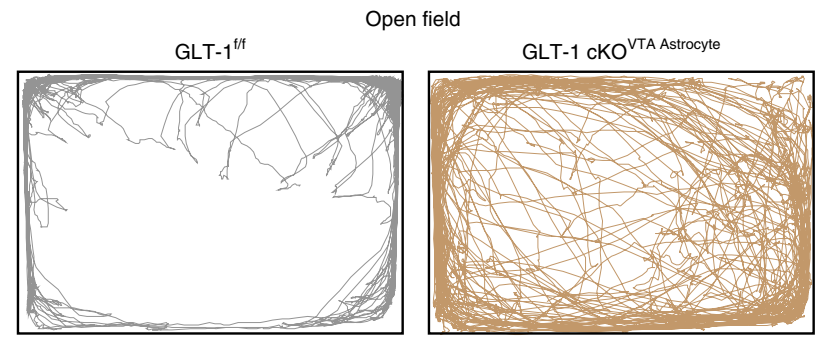

c

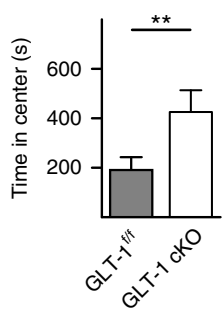

d

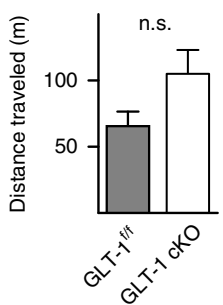

Fig. 8 GLT-1 removal from VTA astrocytes disrupts endogenous avoidance behavior. a Schematic depicting experimental design. b Track plots from a GLT$1^{\mathrm{f} / \mathrm{f}}$ mouse with only a fluorescent reporter (eYFP) on astrocytes displaying normal thigmotaxis in an open field (left), and a GLT-1 cKOVTA Astrocyte (GLT-1 $1^{f / f}$, gfaABC1D::CreVTA) mouse with increased tendency to explore the center of the open field (right). c, $\mathbf{d}$ GLT-1 cKOVTA Astrocyte mice spent more time exploring the center of the open field without affecting locomotor activity (d; unpaired $t$-test $t_{(16)}=1.981, P=0.065, n=9$ mice). ${ }^{\star \star} p<0.01$; Error bars indicate \pm SEM

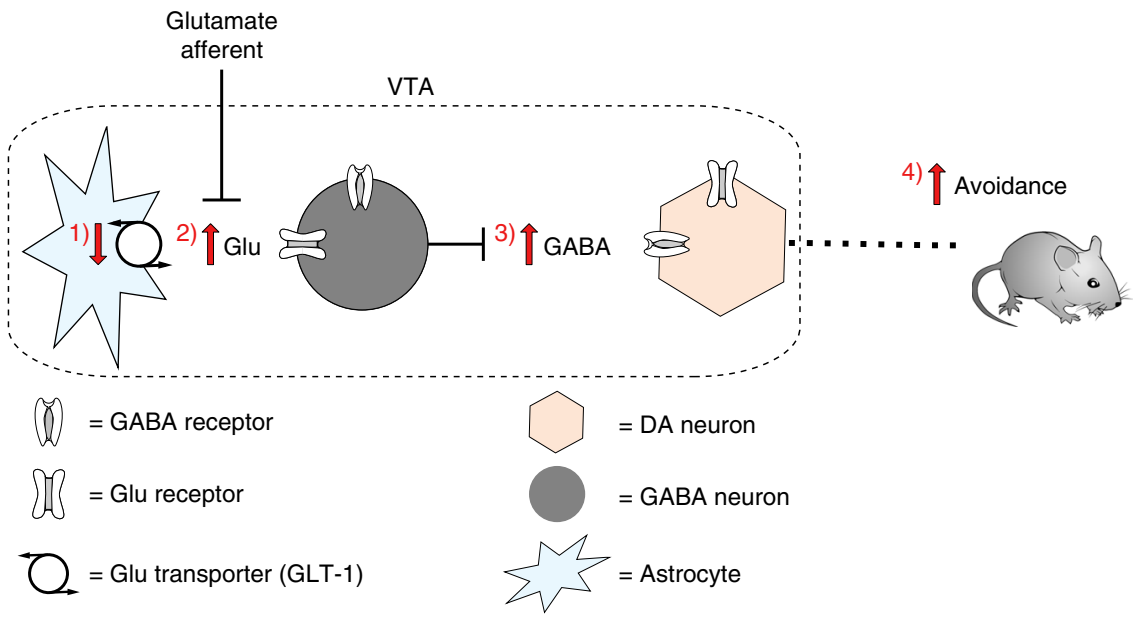

Fig. 9 A neuron-astrocyte circuit for avoidance. In a sequence of events, (1) reduction of glutamate uptake by the astrocytic glutamate transporter, GLT-1, leads to (2) increased glutamate concentration at synapses on VTA GABA neurons. (3) The resultant increase in GABA release at synapses on DA neurons inhibits DA neuron activity, eliciting (4) real-time and learned avoidance behavior 
astrocytes have the capacity to drive behavior, and not merely modulate the gain or polarity of an ongoing behavior. They underscore a computational role for astrocytes in the VTA network, and provide the first evidence that VTA astrocytes can selectively and reliably determine complex motivated behavior.

The complexity of afferents arriving at the VTA points to its importance as a nexus where signals related to sensory experience, valence, and behavioral state intersect with associative processes to cue motivated behavior. For example, separate projections from the bed nucleus of the stria terminalis (BNST) to the VTA mediate both aversive and rewarding signals that promote and attenuate anxiety phenotypes, respectively ${ }^{42}$. But, the BNST provides equivalent projections to both GABA and dopamine neurons within the VTA ${ }^{43}$. Afferents from the pedunculopontine nucleus to both VTA GABA and dopamine neurons mediate approach behaviors like conditioned place preference for reward 44,45 . The VTA is therefore capable of parsing avoidance signals from different afferents to drive avoidance behavior and appropriately coordinate approach and avoidance. But a distinct circuit for avoidance capable of sufficiently opposing approach had not been described.

Inhibition exerts a powerful regulatory influence on dopamine neurons ${ }^{46}$, and prior studies establish that direct depolarization of VTA GABA neurons elicits real-time avoidance but fails to disrupt conditioned responses to reward-predictive cues ${ }^{6,7}$. Our experiments reliably reproduce the real-time avoidance results solely through optogenetic activation of VTA astrocytes. Furthermore, they illustrate that a single astrocytic mechanism can facilitate dopamine neuron inhibition, induces both active and learned avoidance, and interferes with expression of cocaine CPP. All rely on GLT-1 expression at VTA astrocytes; when VTA astrocytes lack functional GLT-1, cued conditioning fails to produce learned avoidance, and also fails to block expression of cocaine CPP (Fig. 7g, h). The selective influence of astrocyte mechanisms on avoidance but not approach in the dopaminergic VTA circuit reinforces the view that the microcircuits mediating avoidance and approach are functionally separate within the VTA, but converge at the level of inputs to dopamine neurons 9 .

Our results demonstrate that VTA astrocytes can exert opposing effects on GABA and dopamine neurons through serial excitation of GABA neurons and subsequent inhibition of dopamine neurons (Fig. 9). The unexpected lack of direct astrocyte-mediated glutamatergic effects at dopamine neurons concurrent with those observed at GABA neurons highlight the separate circuits for avoidance and approach, and may reflect a partitioning mechanism akin to the basal ganglia wherein distinct subpopulations of astrocytes are functionally linked to either D1-type or D2-type receptor-expressing populations of spiny projection neurons ${ }^{17}$ (but see also ref. ${ }^{47}$ ). One possibility is that astrocytic glutamate transport is differentially recruited by GABA and dopamine neurons in the VTA. Astrocyte populations that express distinct complements of transporters and receptors have been reported in the hippocampus ${ }^{16,48}$, and may similarly tile the VTA to partition neuron types and their synapses. Additionally, GLT-1 is expressed in both astrocytes and neurons ${ }^{49}$. Conditional genetic deletion of GLT-1 from neurons blunts the effects of amphetamine, and, in direct contrast to the VTA astrocyte GLT-1 knockout (Fig. 8), has no effect on open field behavior ${ }^{50}$. This indicates that, at least for our open field test results, any potential reduction in neuronal GLT-1 expression due to low-level leak expression of gfaABC1D-driven Cre in neurons would likely not affect our results. Characterizing the molecular diversity, morphology and distribution of astrocytes within the VTA will be required to fully mine the dimensions of their computational capacity ${ }^{51}$.
Astrocytes discriminate the activity of discrete synapses originating from different afferents ${ }^{14,15,20}$. Recent work points to the unique molecular and physiological profiles of ventral midbrain astrocytes relative to their telencephalic counterparts ${ }^{51}$, highlighting the need for approaches tailored to suit the functional dynamics of the system under study ${ }^{52}$. With this in mind, we implemented an astrocyte manipulation protocol aligned to the temporal dynamics of VTA dopamine neurons (where bursting occurs on the order of hundreds of milliseconds) and associated behaviors. Expression of ChR2 specifically in VTA astrocytes exploits the tight association between astrocyte cation permeability and GLT-1 function ${ }^{25-30,35,37}$. By engaging chiefly ion gradients, optical stimulation recruits native astrocyte machinery in electrochemically realistic processes. This approach succeeds in expressing astrocyte-specific, temporally controlled dynamics that make few assumptions about the relatively unknown cellular processes of VTA astrocytes ${ }^{34}$. Finally, the use of visible light wavelengths minimizes reactive gliosis, which often confounds modulatory dynamics with long-lasting inflammatory processes $^{53}$. It is notable that in vivo astrocyte manipulation produced real-time aversion that was readily reversed and spatially manipulated (Fig. 6b, c), signifying that ChR2 activation of astrocytes reflects dynamic modulation rather than long-lasting reactive processes. Optogenetic manipulation of astrocytes also affects only those afferents that are active during ChR2 photoactivation (Fig. 2, 3, and Supplementary Fig. 8) ${ }^{14}$. Therefore, during our conditioned place paradigms, only those neuronal inputs that are active during the paired conditioning session will be facilitated during laser activation.

As a core symptom of anxiety disorders, maladaptive avoidance is considered to be an underlying mechanism maintaining anxiety ${ }^{1}$. Avoidance is a decision to refrain from potential negative outcomes, which could come at the expense of potential rewards. It is in this context that our finding is most relevant; VTA astrocyte GLT-1 can be manipulated to change inhibitory dynamics on dopamine neurons that interfere with the expression of conditioned place preference for reward. It is significant that the conditional loss of GLT-1 eradicates dynamical control of inhibition in the VTA and concurrently reduces avoidance behavior, but preserves approach to reward. Thus, separable pathways within the VTA drive behavioral responses to reward and aversion ${ }^{9}$, and may be subject to distinct pathophysiologies that culminate in maladaptive learning processes ${ }^{1}$. VTA astrocytes may, therefore, reflect a specific target for therapeutics, which have the added advantage of producing effects limited to activated synapses.

Our results establish a previously unknown, intra-VTA neuron-astrocyte circuit mechanism specific to avoidance behavior through which astrocytes exert fast, dynamic control of dopamine neuron firing by modulating afferent glutamatergic drive on GABA neurons (glutamatergic afferents $\rightarrow$ astrocyte GLT- $\rightarrow$ GABA neuron EPSP $\rightarrow$ dopamine neuron IPSP $\rightarrow$ avoidance; Fig. 9). We conclude that VTA astrocytes orchestrate temporal control of dopamine neuron firing, rendering them a decisive element in the circuit computation of avoidance, and promising targets for new therapeutic strategies to intervene in pathological disorders of behavior.

\section{Methods}

Animals. Male and female C57BL/6J mice, Vgat-ires-Cre mice (016962 Stock) and GLT-1 ${ }^{\mathrm{f} / \mathrm{f}}\left(\text { GLT-1 } 1^{\text {flox/flox }}\right)^{39}$ mice were used. GLT-1 ${ }^{\mathrm{f} / \mathrm{f}}$ mice (Slc1A2tm1.1Pros; MGI: 5752263) were obtained from the founder colony at Boston Children's Hospital. All procedures were approved by the University of Texas at San Antonio Institutional Animal care and Use Committees in accordance with the National Institutes of Health guidelines. 
Creation of AAV-TAS-gfaABC1D-ChR2-EYFP. With the creation of an abbreviated GFAP promoter that maintained astrocyte specificity ${ }^{38}$, expression is amplified by using a transcriptional amplification strategy (TAS). This includes cloning a mini-CMV promoter driving expression of Gal4 binding domain fused with the p65 transcriptional activation domain on the opposite strand, and 5 concatenated Gal4 binding sites between the two promoters, mini-CMV and gfaABC1D ${ }^{54}$. Using PCR, the TAS-gfaABC1D enhancer and promoter was amplified and isolated with AscI/SalI sites on the 5' and 3' ends, respectively. This fragment was subcloned into a AAV-CaMKII-ChR2-EYFP by digesting the plasmid with MluI/SalI to remove the CaMKII promoter and have compatible cohesive ends with the new promoter. The AAV vector was packaged and pseudo-typed with AAV5 at the UNC vector core.

Stereotaxic injections and fiber optic implantations. 8 to 12 week old mice were anesthetized using isoflurane anesthesia. Stereotaxic injections of different viruses $(250 \mathrm{~nL}$ at a rate of $100 \mathrm{nl} / \mathrm{min}$ ) were delivered to the VTA using the following coordinates relative to bregma AP: -2.6 to $-3.2 \mathrm{ML}: \pm 0.6 \mathrm{DV}:-4.5$. Mice received either AAV5/TAS-gfaABC1D-ChR2-eYFP or AAV5-GFA104-eGFP. Vgat-ires-Cre mice received injections of AAV5/gfaABC1D-ChR2-eYFP and AAV5/EF1 $\alpha$-DIOeNpHR3.0-eYFP. GLT-1 ${ }^{\mathrm{f} / \mathrm{f}}$ mice received injections of AAV5/gfaABC1D-ChR2eYFP only or AAV5/gfaABC1D-ChR2-eYFP with AAV-gfaABC1D-Cre.

All mice were implanted with custom-made fiber optic cannulas $100 \mu \mathrm{m}$ above the injection site. Custom-made cannulas were constructed using $1.25 \mathrm{~mm}$ diameter ferrule, $200 \mu \mathrm{m}$ fiber optic cable with NA 0.39 and a two-part epoxy. The fiber optic cannulas were secured to the skull of each mouse using miniature screws and dental cement. Mice were singly housed and allowed to recover for 12 days before behavioral experiments were performed. A total of 3 mice were excluded from analysis for having virus and/or fiber optics outside of the VTA.

Immunohistochemistry. Once electrophysiology recordings were completed, the slices were incubated in $4 \%$ paraformaldehyde (PFA) for $30 \mathrm{~min}$ and moved to phosphate buffered solution (PBS) with $.02 \%$ sodium azide for long-term storage at $4{ }^{\circ} \mathrm{C}$.

Mice used for behavioral experiments were anesthetized and intracardially perfused with ice-cold PBS, followed by $4 \%$ PFA. The brains were removed and placed in $4 \%$ PFA overnight at $4{ }^{\circ} \mathrm{C}$. The brains were next rinsed with PBS and 100 $\mu \mathrm{M}$ coronal slices containing VTA were collected. The slices were placed in PBS with $.02 \%$ sodium azide for long-term storage at $4{ }^{\circ} \mathrm{C}$.

On day one slices were rinsed twice with PBS for $10 \mathrm{~min}$. Slices were blocked and permeabilized using $5 \%$ normal goat serum (NGS) $/ 0.2 \%$ triton X-100 in PBS for $2 \mathrm{~h}$ at room temperature. Slices were incubated overnight at $4{ }^{\circ} \mathrm{C}$ with the following primary antibodies: Rabbit anti NeuN (1:1000, ABCAM), chicken anti tyrosine hydroxylase (1:500, AB9702), rabbit anti EAAT-2 (1:500, AB41621), mouse anti GABA (A0310) in $1 \%$ normal goat serum $/ 0.1 \%$ triton X-100 with PBS. On day two, slices were washed three times, for $10 \mathrm{~min}$ with $\mathrm{PBS} / 0.05 \%$ triton X100. Afterwards the slices were incubated with the following secondary antibodies: goat anti-rabbit Alexa 488 (1:1000, A11034) or Alexa 546 (1:1000, A11035), goat anti-chicken Alexa 488 (1:1000, A11039), Alexa 546 (1:1000, A11040) or 647 $(1: 1000, \mathrm{~A} 21449)$ in $1 \%$ normal goat serum $/ 0.1 \%$ triton X-100 in PBS for $2 \mathrm{~h}$ at room temperature. Finally, the slices were washed three times for $10 \mathrm{~min}$ with PBS/ $0.05 \%$ triton $\mathrm{X}-100$ at room temperature and mounted onto slides using Fluoromount-G (0100-20). Bright field and fluorescence images for fiber and opsin placement were obtained using a CCD camera on a fluorescent stereoscope. Sections containing the ventral midbrain were imaged using a Zeiss confocal at $\times 4$ and $\times 20$ magnification with $1.0 \mu \mathrm{m}$ thick optical sections. Each confocal image was obtained using identical settings for gain, laser and pinhole size. Images were then analyzed using ImageJ FIJI software.

Flow cytometry. The VTA was carefully sectioned from GLT-1 $1^{\mathrm{f} / \mathrm{f}}$ and $\mathrm{cKO}$ mice in $1 \mathrm{X}$ Hanks's Balanced Salt Solution (HBSS). The tissue was minced and incubated in pronase and repeatedly triturated every $20 \mathrm{~min}$ for $1 \mathrm{~h}$. DNASE $(100 \mathrm{units} / \mathrm{ml}$, Worthington) was added to the HBSS containing pronase solution to prevent cell aggregation. After $1 \mathrm{~h}$ the cell suspension was diluted with $1 \times$ HBSS and centrifuged at $500 \times g$ for $5 \mathrm{~min}$. The pellet was resuspended in HBSS and filtered through a $100 \mu \mathrm{m}$ filter. The cells were counted, and the filtrate was centrifuged and resuspended in Phosphate Buffer Saline (PBS).

The cells were resuspended in $4 \%$ paraformaldehyde (PFA; Sigma) for $15 \mathrm{~min}$ and subsequently washed with $100 \mathrm{mM}$ glycine (Sigma) in PBS to remove any traces of PFA. After centrifugation, the cells were resuspended in 5\% donkey serum in $0.3 \%$ Triton-X for 30 min to block and permeabilize the cell membrane. The cells were then incubated with primary antibodies: mouse anti- GFAP (1:1000, G3893), rabbit anti EEAT-2 (1:1000, AB41621) followed by secondary antibodies: donkey anti mouse Alexa 488 (1:1000, A21202), donkey anti-rabbit Alexa 555 (1:1000, A31572) for 20 and $15 \mathrm{~min}$, respectively. Cells were analyzed in a BD LSR II flow equipped with three excitation lasers $(488,555$, and $633 \mathrm{~nm})$. Briefly, events were selected based on their scatter properties indicative of cells, then selected for single cells, and selected based on fluorescence intensities. All gates were determined based on unstained, single-color and fluorescent minus 1 GLT- $1^{\mathrm{f} / \mathrm{f}}$ of mouse brain cells. Gating analysis was done using FlowJo software (Treestar).
Open field test. Mice were placed in a custom-made plastic rectangular box $(50 \times$ $30 \mathrm{~cm}$ ) and the box was divided into a center and outer zone with the outer zone being $7 \mathrm{~cm}$ from the wall. Mice were allowed to freely explore for $30 \mathrm{~min}$. Anymaze (Stoelting) video tracking system was used to track the distance each mouse traveled and how much time they spent in each zone for all behavioral experiments.

Real-time place preference (RTPP). Mice were placed in a custom-made plastic rectangular box $(50 \mathrm{~cm} \times 30 \mathrm{~cm})$ with no distinguishing cues. One side of the chamber was randomly designated to be the side where the lasers would be activated. Individual mice were initially placed in the chamber and allowed to explore before beginning the experiment. During the 30-minute session the laser would be activated ( $1 \mathrm{~Hz}: 0.5 \mathrm{~s}$ pulse duration, light intensity between 15 and $20 \mathrm{~mW}$ ) each time the mouse entered the laser-paired side of the chamber, and would be turned off when the mouse crossed back into the non-stimulation side of the chamber. The difference score was calculated by taking the time the mouse spent in the Laser side minus time in the No Laser side.

Mice expressing ChR2 or eYFP in astrocytes were connected to a $473 \mathrm{~nm}$ laser using a $1 \times 1$ rotary joint. For mice expressing ChR2 in astrocytes and NpHR3.0 in GABA cells, we used a $2 \times 1$ rotary joint connected to $405 \mathrm{~nm}$ and $593 \mathrm{~nm}$ laser. This allowed us to activate either ChR2 or NpHR3.0 individually or together. The same set up was used for all RTPP experiments.

Laser conditioned place avoidance (Laser CPA). Laser CPA experiments were performed over a four-day period using a two-chamber conditioning apparatus (Med Associates) with each chamber consisting of a black chamber with a vertical metal bar floor, and a white chamber with a mesh floor. One mouse with a strong initial preference for either chamber (defined as a mouse spending more than $75 \%$ of the time in either chamber) was discarded from analysis. On day 1 (pre-test), mice were placed in one of the two sides and allowed to freely explore the entire apparatus for $30 \mathrm{~min}$. During days 2 and 3 (conditioning) mice were confined to one of the two chambers (counterbalanced across all mice) and received optical stimulation $(0.5 \mathrm{sec}$ pulse, $18 \mathrm{~mW}$ ) once a minute for $30 \mathrm{~min}$, or no stimulation. During the no stimulation session mice were connected the same as during the laser session, but the laser was off. A minimum of $4 \mathrm{~h}$ after the first training session mice were placed into the other side of the chamber and the treatment reversed. Day 4 (post-test), mice were allowed to freely roam the apparatus for $30 \mathrm{~min}$. The difference score was calculated by taking the time each mouse spent on the laser-paired side in the post-test minus the time spent in the pre-test.

Cocaine conditioned place preference (cocaine CPP). A modified version of Laser CPA and the same chamber were used to perform cocaine CPP experiments. The pre-test and the post-test were performed identically, but the conditioning sessions were changed. During days 2 and 3 of conditioning, mice were confined to one of the two chambers (counterbalanced across all mice) and received an i.p. cocaine $(15 \mathrm{mg} / \mathrm{kg})$ injection paired with optical stimulation $(0.5 \mathrm{sec}$ pulse, $18 \mathrm{~mW})$ once a minute for $30 \mathrm{~min}$, or i.p. saline injection with no laser stimulation. During both conditioning sessions mice were tethered to the patch cable. The difference score was calculated by taking the time each mouse spent on the cocaine/laserpaired side in the post-test minus the time spent in the pre-test.

Brain slice preparation and electrophysiology. After completion of behavioral experiments, adult 10 to 14 week old mice were deeply anesthetized with isoflurane followed by rapid decapitation. The brain was quickly removed and chilled in icecold cutting solution containing (in mM): 110 cholineCl, $2.5 \mathrm{KCl}, 1.25 \mathrm{NaH}_{2} \mathrm{PO}_{4}$, $7 \mathrm{MgCl}_{2}, 0.5 \mathrm{CaCl}_{2}, 10$ dextrose, $25 \mathrm{NaHCO}_{3}$, and oxygenated with $95 \% \mathrm{O} 2-5 \%$ $\mathrm{CO}_{2} .250 \mu \mathrm{m}$ horizontal brain slices containing VTA were incubated for $30 \mathrm{~min}$ at $35^{\circ} \mathrm{C}$ in a chamber filled with artificial cerebrospinal fluid (aCSF) and stored at room temperature for the remainder of the day. Slices were transferred to a recording chamber where they continuously received $\operatorname{aCSF}\left(33-35^{\circ} \mathrm{C}\right)$ at a rate of $2 \mathrm{ml}$ per minute by a gravity-feed system. aCSF was composed of (in $\mathrm{mM}$ ): $126 \mathrm{NaCl}, 2.5 \mathrm{KCl}, 1.25 \mathrm{NaH}_{2} \mathrm{PO}_{4}, 2 \mathrm{MgCl}_{2}, 2 \mathrm{CaCl}_{2}, 10$ dextrose, $25 \mathrm{NaHCO}_{3}$, was equilibrated using $95 \% \mathrm{O}_{2}-5 \% \mathrm{CO}_{2}$ and had a $\mathrm{pH}$ of 7.2 .

Midbrain neurons and astrocytes were visualized using gradient contrast illumination through a $\times 40$ water-immersion lens attached to an Olympus BX51 upright microscope. The VTA was defined as neurons medial to the medial terminal nucleus of the accessory optic tract. Neurons were considered dopaminergic based on the following electrophysiological criteria: have a hyperpolarization-activated inward current, slow spontaneous firing frequency $\leq 5 \mathrm{~Hz}$ and an action potential half-width $\geq 1.5 \mathrm{~ms}$. Neurons were considered GABAergic based on the following electrophysiological criteria: continuous fast firing without spike frequency accommodation upon depolarizing current injection, fast spontaneous firing frequency $>10 \mathrm{~Hz}$ and an action potential halfwidth $\leq 1.0 \mathrm{~ms}$. Dopamine (TH-positive) and GABA (GABA-positive) neurons filled with biocytin were further identified immunocytochemically

(Supplementary Fig. 4). Astrocytes expressed GFP or eYFP and were visualized with a $\times 40$ or $\times 60$ water-immersion objective on a fluorescent microscope capable of detecting GFP/eYFP.

Whole-cell recording pipettes with a tip resistance between 4 and $6 \mathrm{MOhm}$ were pulled from borosilicate glass using a P-97 Flaming/Brown electrode puller 
(Sutter Instruments). For voltage-clamp experiments recording pipettes were filled with internal solution containing (in mM): $110 \mathrm{~d}$-gluconic acid, $110 \mathrm{CsOH}$, $10 \mathrm{CsCl}_{2}, 1$ EGTA, $10 \mathrm{HEPES}, 1 \mathrm{ATP}, 10 \mathrm{~mm}$ phosphocreatine. Pipettes used for current clamp recordings contained (in $\mathrm{mM}$ ): $138 \mathrm{~K}$-gluconate, 10 HEPES, 2 $\mathrm{MgCl}_{2}, 0.2$ EGTA, $0.0001 \mathrm{CaCl}_{2}$, $4 \mathrm{Na}$-ATP, $0.4 \mathrm{Na}-\mathrm{GTP}$.

Postsynaptic currents (PSC) from excitatory and inhibitory afferents were evoked with no blockers present by placing a $250 \mu \mathrm{m}$ bipolar stimulating electrode within $500 \mu \mathrm{m}$ of the neuron being recorded and applying a single pulse $(0.05 \mathrm{~ms})$. PSCs were isolated by voltage clamping the cells at $-55 \mathrm{mV}$ for EPSCs and at +10 $\mathrm{mV}$ for IPSCs. The effect astrocytes had on synaptic activity was tested by photoactivating $\mathrm{ChR} 2$ on astrocytes for $500 \mathrm{~ms}$ (constant stimulation) and evoking PSCs $400 \mathrm{~ms}$ into the optical stimulation period. ChR2 on astrocytes was stimulated using a 473 or $405 \mathrm{~nm}$ laser $(10-20 \mathrm{~mW})$. Afferent-mediated currents on astrocytes were obtained by applying $50 \mathrm{~Hz}$ electrical stimulation (20 pulses, $0.05 \mathrm{~ms}$ pulse width). Whole-cell recordings were made using a Multiclamp 700B amplifier (Molecular Devices). Signals were digitized at $50 \mathrm{kHz}$ and saved to a hard drive for analysis using Axograph X (Axograph).

Statistics. A student's two-tailed $t$-test was used when comparing between two groups. A One-way ANOVA with Tukey's post-hoc test was used for comparisons across multiple groups. A $P$-value $<0.05$ was used as our significance level for all tests. Power analysis showed greater than $80 \%$ power in all analyses.

Reporting summary. Further information on experimental design is available in the Nature Research Reporting Summary linked to this article.

\section{Data availability}

The data supporting these findings are available from the corresponding author upon reasonable request.

Received: 27 August 2018 Accepted: 22 February 2019

Published online: 29 March 2019

\section{References}

1. Mkrtchian, A., Aylward, J., Dayan, P., Roiser, J. P. \& Robinson, O. J. Modeling avoidance in mood and anxiety disorders using reinforcement learning. Biol. Psychiatry 82, 532-539 (2017).

2. McCullough, L. D., Sokolowski, J. D. \& Salamone, J. D. A neurochemical and behavioral investigation of the involvement of nucleus accumbens dopamine in instrumental avoidance. Neuroscience 52, 919-925 (1993).

3. Lammel, S., Ion, D. I., Roeper, J. \& Malenka, R. C. Projection-specific modulation of dopamine neuron synapses by aversive and rewarding stimuli. Neuron 70, 855-862 (2011).

4. Tian, J. et al. Distributed and mixed information in monosynaptic inputs to dopamine neurons. Neuron 91, 1374-1389 (2016).

5. Ungless, M. A., Magill, P. J. \& Bolam, J. P. Uniform inhibition of dopamine neurons in the ventral tegmental area by aversive stimuli. Science 303, 2040-2042 (2004).

6. Tan, K. R. et al. GABA neurons of the VTA drive conditioned place aversion. Neuron 73, 1173-1183 (2012).

7. van Zessen, R., Phillips, J. L., Budygin, E. A. \& Stuber, G. D. Activation of VTA GABA neurons disrupts reward consumption. Neuron 73, 1184-1194 (2012).

8. Chang, C. Y. et al. Brief optogenetic inhibition of dopamine neurons mimics endogenous negative reward prediction errors. Nat. Neurosci. 19, 111-116 (2016).

9. Matsumoto, H., Tian, J., Uchida, N. \& Watabe-Uchida, M. Midbrain dopamine neurons signal aversion in a reward-context-dependent manner. Elife 5, pii: e17328 (2016).

10. Oliet, S. H., Piet, R. \& Poulain, D. A. Control of glutamate clearance and synaptic efficacy by glial coverage of neurons. Science 292, 923-926 (2001)

11. Sonnewald, U., Westergaard, N. \& Schousboe, A. Glutamate transport and metabolism in astrocytes. Glia 21, 56-63 (1997).

12. Chatton, J. Y., Marquet, P. \& Magistretti, P. J. A quantitative analysis of Lglutamate-regulated $\mathrm{Na}+$ dynamics in mouse cortical astrocytes: implications for cellular bioenergetics. Eur. J. Neurosci. 12, 3843-3853 (2000).

13. Devaraju, P., Sun, M. Y., Myers, T. L., Lauderdale, K. \& Fiacco, T. A. Astrocytic group I mGluR-dependent potentiation of astrocytic glutamate and potassium uptake. J. Neurophysiol. 109, 2404-2414 (2013).

14. Armbruster, M., Hanson, E. \& Dulla, C. G. Glutamate clearance is locally modulated by presynaptic neuronal activity in the cerebral cortex. J. Neurosci. 36, 10404-10415 (2016).

15. Perea, G. \& Araque, A. Properties of synaptically evoked astrocyte calcium signal reveal synaptic information processing by astrocytes. J. Neurosci. 25, 2192-2203 (2005).
16. Khakh, B. S. \& Sofroniew, M. V. Diversity of astrocyte functions and phenotypes in neural circuits. Nat. Neurosci. 18, 942-952 (2015).

17. Martin, R., Bajo-Graneras, R., Moratalla, R., Perea, G. \& Araque, A. Circuitspecific signaling in astrocyte-neuron networks in basal ganglia pathways. Science 349, 730-734 (2015).

18. Nieh, E. H. et al. Inhibitory input from the lateral hypothalamus to the ventral tegmental area disinhibits dopamine neurons and promotes behavioral activation. Neuron 90, 1286-1298 (2016).

19. Martin-Fernandez, M. et al. Synapse-specific astrocyte gating of amygdalarelated behavior. Nat. Neurosci. 20, 1540-1548 (2017).

20. Covelo, A. \& Araque, A. Neuronal activity determines distinct gliotransmitter release from a single astrocyte. Elife 7, pii: e32237 (2018).

21. Mudrick-Donnon, L. A., Williams, P. J., Pittman, Q. J. \& MacVicar, B. A. Postsynaptic potentials mediated by GABA and dopamine evoked in stellate glial cells of the pituitary pars intermedia. J. Neurosci. 13, 4660-4668 (1993).

22. Mennerick, S., Benz, A. \& Zorumski, C. F. Components of glial responses to exogenous and synaptic glutamate in rat hippocampal microcultures. J. Neurosci. 16, 55-64 (1996).

23. Porter, J. T. \& McCarthy, K. D. Hippocampal astrocytes in situ respond to glutamate released from synaptic terminals. J. Neurosci. 16, 5073-5081 (1996).

24. Clark, B. A. \& Barbour, B. Currents evoked in Bergmann glial cells by parallel fibre stimulation in rat cerebellar slices. J. Physiol. 502(Pt 2), 335-350 (1997).

25. Meeks, J. P. \& Mennerick, S. Astrocyte membrane responses and potassium accumulation during neuronal activity. Hippocampus 17, 1100-1108 (2007)

26. Kirischuk, S., Parpura, V. \& Verkhratsky, A. Sodium dynamics: another key to astroglial excitability? Trends Neurosci. 35, 497-506 (2012).

27. Parpura, V. \& Verkhratsky, A. Homeostatic function of astrocytes: $\mathrm{Ca}(2+)$ and $\mathrm{Na}(+)$ signalling. Transl. Neurosci. 3, 334-344 (2012).

28. Unichenko, P., Myakhar, O. \& Kirischuk, S. Intracellular Na+ concentration influences short-term plasticity of glutamate transporter-mediated currents in neocortical astrocytes. Glia 60, 605-614 (2012).

29. Wadiche, J. I., Tzingounis, A. V. \& Jahr, C. E. Intrinsic kinetics determine the time course of neuronal synaptic transporter currents. Proc. Natl Acad. Sci. USA 103, 1083-1087 (2006)

30. Kirischuk, S., Kettenmann, H. \& Verkhratsky, A. Membrane currents and cytoplasmic sodium transients generated by glutamate transport in Bergmann glial cells. Pflug. Arch. 454, 245-252 (2007).

31. Wadiche, J. I., Arriza, J. L., Amara, S. G. \& Kavanaugh, M. P. Kinetics of a human glutamate transporter. Neuron 14, 1019-1027 (1995).

32. Diamond, J. S. \& Jahr, C. E. Transporters buffer synaptically released glutamate on a submillisecond time scale. J. Neurosci. 17, 4672-4687 (1997).

33. Huang, Y. H. \& Bergles, D. E. Glutamate transporters bring competition to the synapse. Curr. Opin. Neurobiol. 14, 346-352 (2004).

34. Perea, G., Yang, A., Boyden, E. S. \& Sur, M. Optogenetic astrocyte activation modulates response selectivity of visual cortex neurons in vivo. Nat. Commun. 5, 3262 (2014).

35. Nagel, G. et al. Channelrhodopsin-2, a directly light-gated cationselective membrane channel. Proc. Natl. Acad. Sci. USA 100, 13940-13945 (2003).

36. Yang, J. et al. $\mathrm{Na}(+)-\mathrm{Ca}(2)(+)$ exchanger mediates $\mathrm{ChR} 2$-induced $[\mathrm{Ca}(2)(+)] \mathrm{i}$ elevation in astrocytes. Cell Calcium 58, 307-316 (2015).

37. Sasaki, T. et al. Application of an optogenetic byway for perturbing neuronal activity via glial photostimulation. Proc. Natl. Acad. Sci. USA 109, 20720-20725 (2012).

38. Lee, Y., Messing, A., Su, M. \& Brenner, M. GFAP promoter elements required for region-specific and astrocyte-specific expression. Glia 56, 481-493 (2008).

39. Petr, G. T. et al. Conditional deletion of the glutamate transporter GLT-1 reveals that astrocytic GLT-1 protects against fatal epilepsy while neuronal GLT-1 contributes significantly to glutamate uptake into synaptosomes. J. Neurosci. 35, 5187-5201 (2015)

40. Bardo, M. T. \& Bevins, R. A. Conditioned place preference: what does it add to our preclinical understanding of drug reward? Psychopharmacol. (Berl.) 153, 31-43 (2000)

41. Zweifel, L. S., Argilli, E., Bonci, A. \& Palmiter, R. D. Role of NMDA receptors in dopamine neurons for plasticity and addictive behaviors. Neuron 59, 486-496 (2008).

42. Jennings, J. H. et al. Distinct extended amygdala circuits for divergent motivational states. Nature 496, 224-228 (2013).

43. Faget, L. et al. Afferent inputs to neurotransmitter-defined cell types in the ventral tegmental area. Cell Rep. 15, 2796-2808 (2016).

44. Yau, H. J. et al. Pontomesencephalic tegmental afferents to VTA nondopamine neurons are necessary for appetitive pavlovian learning. Cell Rep. 16, 2699-2710 (2016) 
45. Yoo, J. H. et al. Activation of pedunculopontine glutamate neurons is reinforcing. J. Neurosci. 37, 38-46 (2017).

46. Lobb, C. J., Wilson, C. J. \& Paladini, C. A. A dynamic role for GABA receptors on the firing pattern of midbrain dopaminergic neurons. J. Neurophysiol. 104, 403-413 (2010).

47. Octeau, J. C. et al. An optical neuron-astrocyte proximity assay at synaptic distance scales. Neuron 98, 49-66 e49 (2018).

48. Matthias, K. et al. Segregated expression of AMPA-type glutamate receptors and glutamate transporters defines distinct astrocyte populations in the mouse hippocampus. J. Neurosci. 23, 1750-1758 (2003).

49. Chen, W. et al. The glutamate transporter GLT1a is expressed in excitatory axon terminals of mature hippocampal neurons. J. Neurosci. 24, 1136-1148 (2004).

50. Fischer, K. D. et al. Behavioral phenotyping and dopamine dynamics in mice with conditional deletion of the glutamate transporter GLT-1 in neurons: resistance to the acute locomotor effects of amphetamine. Psychopharmacol. (Berl.) 235, 1371-1387 (2018).

51. Xin, W. \& Bonci, A. Functional astrocyte heterogeneity and implications for their role in shaping neurotransmission. Front Cell Neurosci. 12, 141 (2018).

52. Paukert, $\mathrm{M}$. et al. Norepinephrine controls astroglial responsiveness to local circuit activity. Neuron 82, 1263-1270 (2014).

53. Pekny, M. \& Pekna, M. Astrocyte reactivity and reactive astrogliosis: costs and benefits. Physiol. Rev. 94, 1077-1098 (2014).

54. Liu, B., Paton, J. F. \& Kasparov, S. Viral vectors based on bidirectional cellspecific mammalian promoters and transcriptional amplification strategy for use in vitro and in vivo. BMC Biotechnol. 8, 49 (2008).

\section{Acknowledgements}

We thank J. Wadiche, C. Wilson and M. Higgs for helpful comments. Artwork by Victoria Foor (http://victoriafoor.com). Funding: This research was supported by National Institutes of Health Grants MH079276 (C.A.P.), MH113341 (C.A.P.), DA030530 (C.A.P.), DA033386 (M.J.W.), DA042362 (M.J.W.), GM060655 (J.A.G.), F31DA041303 (J.A.G.), NS066019 (P.A.R.), MH104318 (P.A.R.), DA032701 (M.J.B.) and National Science Foundation Grant HRD-1249284 (J.A.G.). Imaging data collection was supported by Grant G12MD007591.

\section{Author contributions}

J.A.G., J.M.P., G.M.B., K.T., A.M.M., M.J.B., C.W.T., and E.A.S. collected data. J.A.G., E.A.S., K.T., A.M.M., J.M.P., M.J.W., M.J.B., C.W.T., and C.A.P. analyzed data. J.A.G., J.M.P., G.M.B., E.A.S., K.T., M.J.B., C.W.T., N.B.C., and P.A.R. prepared animals and reagents. J.A.G., S.A.Q., and C.A.P. wrote the paper.

\section{Additional information}

Supplementary Information accompanies this paper at https://doi.org/10.1038/s41467019-09131-y.

Competing interests: The authors declare no competing interests.

Reprints and permission information is available online at http://npg.nature.com/ reprintsandpermissions/

Journal peer review information: Nature Communications thanks Marta Navarrete and the other anonymous reviewers for their contribution to the peer review of this work.

Publisher's note: Springer Nature remains neutral with regard to jurisdictional claims in published maps and institutional affiliations.

\begin{abstract}
cc (i) Open Access This article is licensed under a Creative Commons Attribution 4.0 International License, which permits use, sharing, adaptation, distribution and reproduction in any medium or format, as long as you give appropriate credit to the original author(s) and the source, provide a link to the Creative Commons license, and indicate if changes were made. The images or other third party material in this article are included in the article's Creative Commons license, unless indicated otherwise in a credit line to the material. If material is not included in the article's Creative Commons license and your intended use is not permitted by statutory regulation or exceeds the permitted use, you will need to obtain permission directly from the copyright holder. To view a copy of this license, visit http://creativecommons.org/ licenses/by/4.0/.
\end{abstract}

(C) The Author(s) 2019 